\title{
Regolith behavior under asteroid-level gravity conditions: low-velocity impact experiments
}

\author{
Julie Brisset* ${ }^{*}$, Joshua Colwell, Adrienne Dove, Sumayya Abukhalil, Christopher Cox and Nadia Mohammed
}

\begin{abstract}
The dusty regolith covering the surfaces of asteroids and planetary satellites differs in size, shape, and composition from terrestrial soil particles and is subject to environmental conditions very different from those found on Earth. This regolith evolves in a low ambient pressure and low-gravity environment. Its response to low-velocity impacts, such as those that may accompany human and robotic exploration activities, may be completely different than what is encountered on Earth. Experimental studies of the response of planetary regolith in the relevant environmental conditions are thus necessary to facilitate future Solar System exploration activities.

We combined the results and provided new data analysis elements for a series of impact experiments into simulated planetary regolith in low-gravity conditions using two experimental setups and a range of microgravity platforms. The Physics of Regolith Impacts in Microgravity Experiment (PRIME) flew on several parabolic aircraft flights, enabling the recording of impacts into granular materials at speeds of $\sim 4-230 \mathrm{~cm} / \mathrm{s}$. The COLLisions Into Dust Experiment (COLLIDE) is conceptually close to the PRIME setup. It flew on the Space Shuttle in 1998 and 2001 and more recently on the Blue Origin New Shepard rocket, recording impacts into simulated regolith at speeds between 1 and $120 \mathrm{~cm} / \mathrm{s}$. Results of these experimental campaigns found that there is a significant change in the regolith behavior with the gravity environment. In a $10^{-2} \mathrm{~g}$ environment (with $\mathrm{g}$ being the gravity acceleration at the surface of the Earth), only embedding of the impactor was observed and ejecta production was produced for most impacts at $>20 \mathrm{~cm} / \mathrm{s}$. Once at microgravity levels $\left(<10^{-4} \mathrm{~g}\right)$, the lowest impact energies also produced impactor rebound. In these microgravity conditions, ejecta started to be produced for impacts at $>10 \mathrm{~cm} / \mathrm{s}$. The measured ejecta speeds were somewhat lower than the ones measured at reduced-gravity levels, but the ejected masses were higher. In general, the mean ejecta velocity shows a power-law dependence on the impact energy with an index of $\sim 0.5$. When projectile rebound occurred, we observed that its coefficients of restitution on the bed of regolith simulant decrease by a factor of 10 with increasing impact speeds from $\sim 5$ up to $100 \mathrm{~cm} / \mathrm{s}$. We could also observe an increased cohesion between the JSC-1 grains compared to the quartz sand targets.
\end{abstract}

Keywords: Regolith, Low-velocity impacts, Microgravity experiments

\section{Introduction}

Small airless bodies of the Solar System are known to be covered in a layer of regolith, composed of grains of varying sizes. Created by the bombardment of micrometeoroids or thermal disintegration, micron-sized and larger dust particles collect on the surfaces of asteroids, comets, and moons (Housen et al. 1979; Housen

*Correspondence: julie.brisset@ucf.edu

Florida Space Institute, University of Central Florida, 12354 Research Parkway,

Partnership 1 Building, Suite 211, Orlando 32826, FL, USA and Wilkening 1982). Any space mission probing or landing on the surfaces of these small bodies has to interact with this regolith, at the very low surface gravity levels induced by the body's small mass. The first soft landings on such small bodies were performed by the NEAR-Shoemaker spacecraft on the asteroid Eros (Veverka et al. 2001), Hayabusa on Itokawa (Kawaguchi et al. 2008), and Philae on the comet 67P/Churyumov-Gerasimenko (Biele et al. 2015). In the latter instances, the landing resulted in an unexpected response of the surface to the low-velocity impact of the lander, in particular the 
remarkable recurrent bouncing of Philae on the comet's surface after a first touchdown at around $1 \mathrm{~m} / \mathrm{s}$.

Currently, two missions are en route to asteroids: Hayabusa-2 carrying the MASCOT lander (Tsuda et al. 2013; Jaumann et al. 2016), due to arrive at Ryugu in 2018, and the OSIRIS-REx mission arriving at Bennu in 2018, planning to sample its surface using a Touch-AndGo mechanism (Lauretta et al. 2017). In addition, the increased interest in In-Situ Resource Utilization (ISRU) and mining prospects will soon lead to more reconnaissance and pathfinder missions to small bodies of the Solar System. Understanding interactions with surface regolith at very low gravity levels has therefore become an imperative. Impacts at a few meters per second down to a few centimeters per second into regolith layers are of particular interest to these current and future missions.

Motivated by these upcoming missions, theoretical and numerical work has recently been performed in order to investigate the behavior of granular material at the surface of small asteroids, such as Ryugu and Bennu (Scheeres et al. 2010; Sanchez and Scheeres 2014; Hirabayashi et al. 2015; Thuillet et al. 2017). In particular, the response of a layer of regolith to a low-velocity impact is investigated in, e.g., Maurel et al. (2017) for MASCOT and Sánchez et al. (2013). Experimental works supporting these efforts are scarce and often performed under conditions very different from what can be expected during a landing/contact at the surface of small bodies (1g, hypervelocity impacts). For example, the experiment campaign performed by Housen and Holsapple (2003) to investigate crater formation in porous materials studied impacts at $>2 \mathrm{~km} / \mathrm{s}$ (see Holsapple et al. (2002) and Housen and Holsapple (2011), for a review of other high-speed impact experiments in porous materials). In order to investigate the relative influence of gravity on the impact cratering and ejecta production processes in very large impact events, Housen et al. (1999) performed high-speed impacts into porous materials in a centrifuge at gravity levels up to $500 \mathrm{~g}$. While these experiments at high impact velocities are relevant for meteorite and interasteroid impacts, the velocity ranges are not applicable to the landing or sample collection on a surface in low gravity.

Theoretical and experimental studies that are more relevant to an asteroid landing situation are found in the study of impactor deceleration in a granular medium: the Katsuragi and Durian $(2007,2013)$ and Katsuragi and Blum (2017) series studies the drag force exerted by a granular material on a penetrating impactor; Clark et al. (2012) and Clark and Behringer (2013) study the impactor stopping time and the energy transfer from the impactor to the granular material at the grain level in quasi2D setups; Seguin et al. (2008) studies the influence of container walls on the impactor penetration depth; and
Machii et al. (2013) measures the influence of the target strength on the impact outcome. All these experiments were performed at impact speeds ranging from a few $10 \mathrm{~cm} / \mathrm{s}$ to a few meters per second and provide therefore relevant data for the exploration of asteroid surfaces. However, their experiments were performed in $1 g$, and the application of their findings to small body surfaces, which can have gravity levels $<10^{-5} g$, remains unexplored.

The study of low-velocity impacts into granular material in reduced gravity was started in 1998 with the Shuttle experiment COLLisions Into Dust Experiment (COLLIDE) (Colwell and Taylor 1999; Colwell 2003). Following experiments were performed during parabolic flight campaigns in 2002 and 2003, using the Physics of Regolith Impacts in Microgravity Experiment (PRIME) hardware setup (Colwell et al. 2008). More recent data collection was obtained with these two experiments during the PRIME-3 (Colwell et al. 2016) and COLLIDE-3 campaigns, on the NASA C-9 aircraft in 2014 and Blue Origin's New Shepard suborbital rocket in 2016, respectively. Both these experiment setups generate impacts of approximately centimeter-sized spherical projectiles onto beds of granular material at speeds of $1-230 \mathrm{~cm} / \mathrm{s}$ and gravity levels ranging from reduced gravity $\left(\sim 10^{-2} g\right)$ to microgravity $\left(<10^{-4} \mathrm{~g}\right)$. The target materials used were quartz sand and JSC-1 lunar and JSC Mars-1 simulants, sieved at particle size $<250 \mu \mathrm{m}$. While the results of each of these experiment campaigns have been published separately (except for the most recent COLLIDE-3 campaign), the collected data set has now reached a size of $\sim 130$ data points and presents the opportunity for a new and combined analysis, with the perspective of applying these experimental results to interactions with the regolith-covered surfaces of small Solar System bodies.

This is the undertaking of the present paper. We combine the data sets of all the COLLIDE and PRIME campaigns and show the collision outcomes, coefficients of restitution, ejecta speeds, and estimated mass in dependance of the impact velocity and environmental $g$-level. "Methods/Experimental" section describes the PRIME and COLLIDE hardware setups and data collected. The "Results" section describes the data analysis and results, which we then discuss in the "Discussion" section.

\section{Methods/Experimental}

The data results presented here were collected over almost two decades during microgravity experimental campaigns on a variety of flight platforms. The hardware used to collect data can be divided into two families: (1) the COLLIDE experiment series, which flew on the Space Shuttle and a suborbital rocket, and (2) the PRIME experiment series, which flew on parabolic aircrafts. 


\section{COLLIDE}

Flight campaigns The first two COLLIDE flight campaigns were performed in 1998 and 2001, onboard the Space Transportation System (Space Shuttle) STS-90 and STS-108 missions, respectively (COLLIDE-1 and COLLIDE-2). The experiment hardware and performance were described in Colwell and Taylor (1999) and Colwell (2003). During these flights, a total of nine impacts into regolith simulant were recorded.

More recently, in April 2016, a modified setup was flown on Blue Origin's New Shepard suborbital rocket (COLLIDE-3). Parts of the Space Shuttle COLLIDE hardware were repurposed for this new campaign, and a total of four impactor boxes were accommodated into the crew capsule of New Shepard. Three of these boxes were similar to the Space Shuttle hardware, launching one projectile into a bed of regolith simulant. The fourth box was outfitted with a multi-launcher instead of the single-launcher configuration, now allowing for three impactors to be sequentially launched onto the bed of regolith simulant during the same experiment run.

Hardware For COLLIDE-3, four individual Impactor Box Systems (IBSs) of COLLIDE-2 were modified for flight on suborbital launch vehicles. We manufactured a vacuum chamber for each IBS in order to perform the impacts under vacuum conditions, because the New Shepard crew capsule stays at atmospheric pressure during the flight. Each vacuum chamber was outfitted with a viewport allowing for a camera outside of the chamber to record the impact. The IBS electronics were re-wired and connected to custom electronics outside the chamber via an electrical feedthrough. Both the chamber and IBS can be seen in Fig. 1.

The camera system was entirely re-designed for the COLLIDE-3 campaign. While COLLIDE-1 and COLLIDE-2 used camcorders recording on digital video tapes, the new design used a GoPro Hero 3+ black edition recording data files on a microSD card. Both configurations required the retrieval of the tapes/memory card after the experiment run.

Three of the IBSs were used as single-launchers (one projectile per experiment run, like in the COLLIDE-1 and COLLIDE-2 campaigns), while the fourth one was modified to allow for the launching of three projectiles during the same experiment run. This modified setup was named multi-launcher. In a single-launcher IBS, the projectile is kept on a compressed spring by an aluminum bar. This bar is held in its closed position by a pin attached to two wires of memory shape alloy. When activated, these wires retract the pin and the released aluminum bar swings in its open position via a rotary spring. More details on the single-launcher setup can be found in Colwell and Taylor (1999) and Colwell (2003).

The multi-launcher is composed of three individual launchers (slightly smaller than a single-launcher), a central stepper motor, and a circular bottom plate. The stepper motor allows for the rotation of the bottom plate, which is outfitted with a drop hole. The projectiles inside individual launchers are kept on a compressed linear spring. They are held in place by an aluminum bar forced in its closed position by the presence of the bottom plate. This bar is held under tension by a rotary spring pressing it towards an open position. When the drop hole aligns
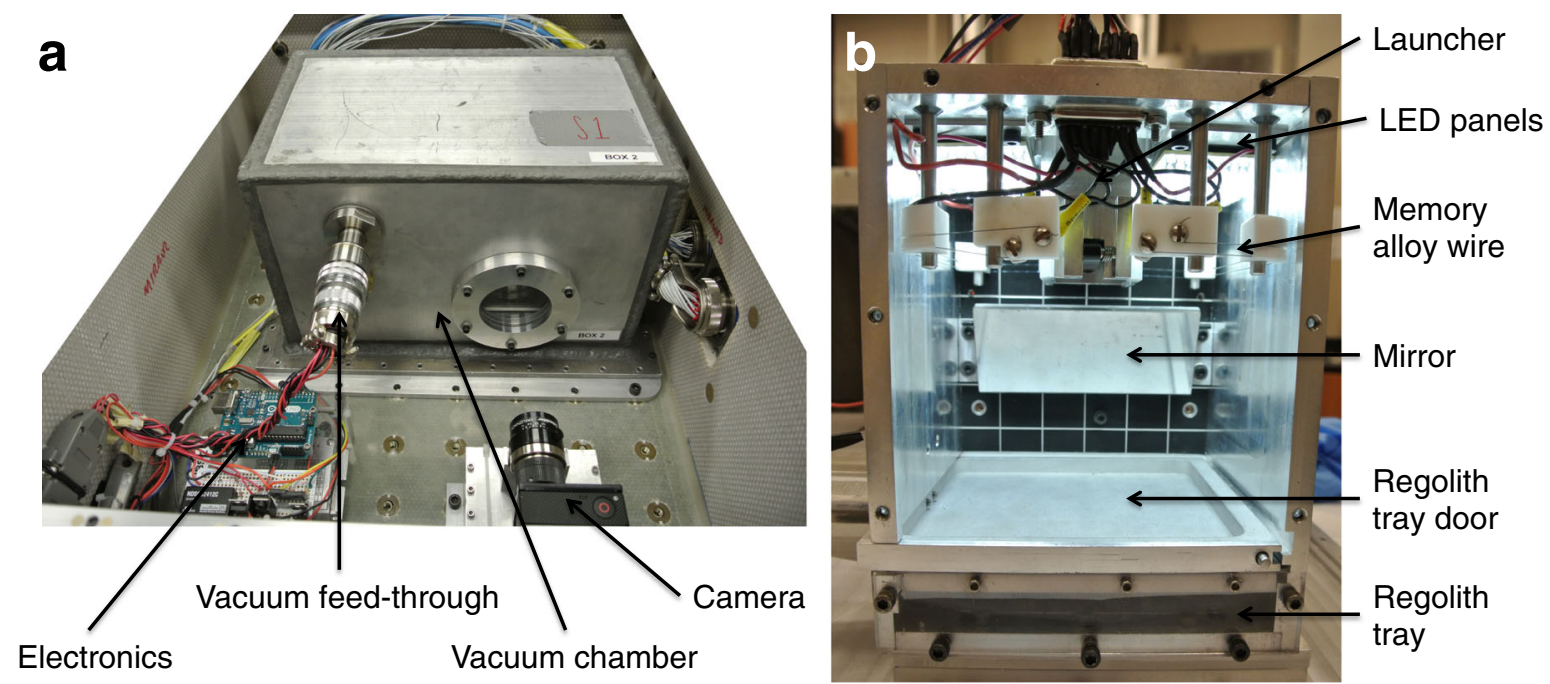

Fig. 1 COLLIDE-3 hardware. a The general setup showing the vacuum chamber with its viewport, the camera for data collection, and the experiment electronics for autonomous experiment run. b The Impactor Box System (IBS) located inside the vacuum chamber with the launcher and regolith tray 
with the launcher, the pin is freed thus allowing for the linear spring of the launcher to push the projectile outwards. Figure 2 shows the multi-launcher setup.

Projectiles and simulants Table 1 details which projectiles and simulants were used for all three experiment campaigns. The projectiles used in the COLLIDE campaigns were Teflon and quartz spheres of diameters between 1 and $2 \mathrm{~cm}$. These projectiles were chosen in order to vary the impact energies over the largest range of values possible while using the same hardware setup. For a similar projectile size, $1 \mathrm{~cm}$ projectiles had masses about one order of magnitude smaller than $2 \mathrm{~cm}$ projectiles.

The target materials used were quartz sand, the lunar mare basalt simulant JSC-1, and the Mars soil simulant JSC Mars-1. The choice of these materials was not motivated by their mineralogic similarity to asteroid regolith, but rather by the shape of their grains. Indeed, from the relatively long history of inter-grain collision experiments (see Blum and Wurm (2008), for a review), it appears that the actual mineral composition of the grains plays a comparatively negligible role in granular material cohesion compared to the size and shape of the grains. A notable exception to this observation takes place if the regolith is composed of ices (in particular water ice) or covered with organics, which are much stickier than rocky regolith and therefore change the behavior of grain layers. Ices and organic materials are outside the scope of the present work, and we therefore only considered grain shape and sizes. The main difference between the chosen simulants is the grain shapes and surface texture, which are increasingly angular and irregular from quartz sand to JSC Mars-1 and to JSC-1 lunar simulants (Fig. 3). Note that quartz sand and JSC Mars-1 are more similar in shape compared with JSC-1.

The target materials were sieved to sizes between 75 and $250 \mu \mathrm{m}$, which provides for a normal distribution between these two sizes, and filled into the target tray of dimensions of $13 \times 13 \times 2 \mathrm{~cm}^{3}$. Grain sizes on the surface of asteroids are known to have a size distribution ranging from micrometers to $10 \mathrm{~m}$. The population of grains smaller than $1 \mathrm{~cm}$ is difficult to infer from available imaging of surfaces, such as for Vesta (Hiroi et al. 1994) and Eros (Dombard et al. 2010), as the image resolutions are usually $>1 \mathrm{~cm}$. However, from thermal inertia measurements, Gundlach and Blum (2013) has inferred average regolith grain sizes covering the surfaces of 26 asteroids. For 15 of these 26 bodies, they determined an average grain size of $<1 \mathrm{~mm}$, which corresponds to the grain size distributions we used in the COLLIDE and PRIME experiments.

Seguin et al. (2008) studied the influence of the grain container size on the penetration depth of projectiles dropped into the container at speeds of $<3 \mathrm{~m} / \mathrm{s}$. They showed that for a granular material target composed of 300-400 $\mu \mathrm{m}$-sized grains and projectile sizes ranging from 5 to $40 \mathrm{~mm}$, the container bottom had an influence on the projectile penetration depth only if located at a few grain diameters from the surface. They also showed that the container side wall location had a vanishing influence on the impactor penetration for a ratio between the tray and projectile of $>5$. Given their impact speeds and the size of their projectiles and target grains, their results are applicable to the COLLIDE and PRIME experiments. As the target trays were $13 \times 13 \mathrm{~cm}^{2}(13>5 \times 2 \mathrm{~cm}$ for a projectile of $2 \mathrm{~cm}$ in diameter) in lateral size and $2 \mathrm{~cm}$
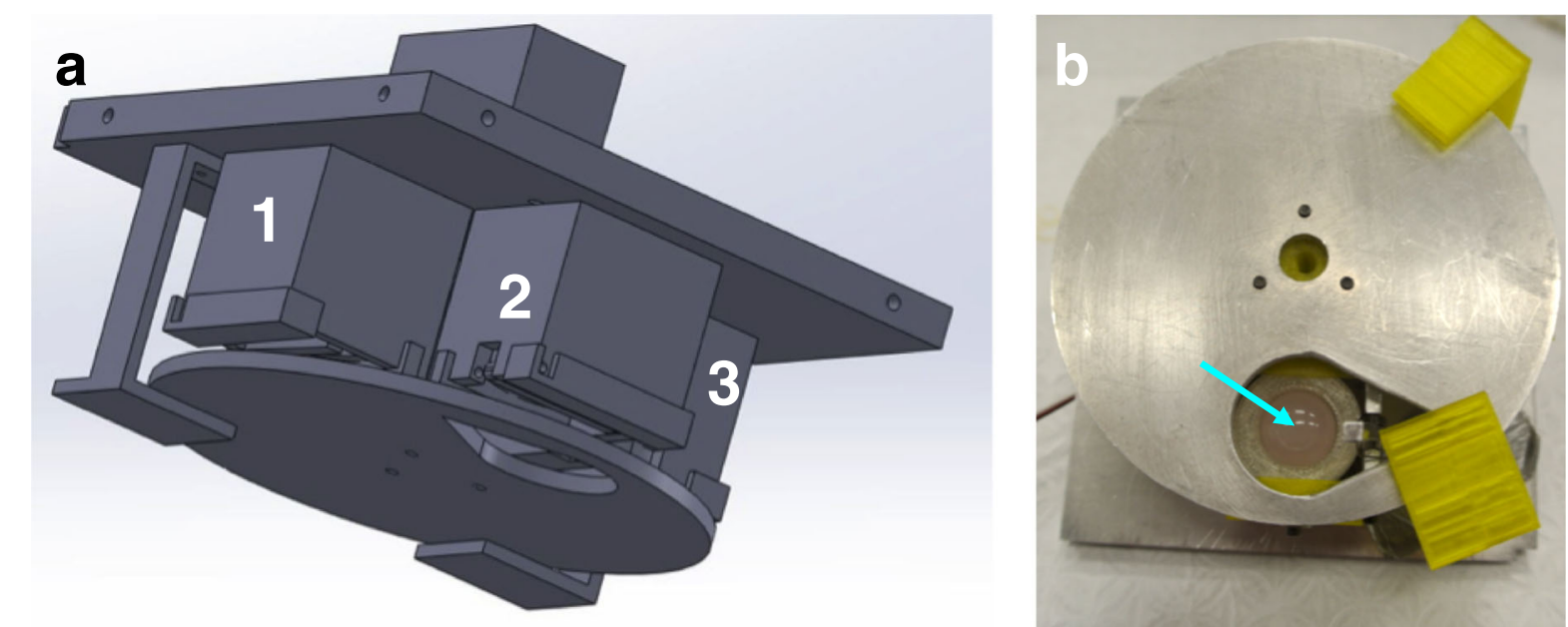

Fig. 2 COLLIDE-3 multi-launcher system. a CAD side view showing the individual launchers (three, numbered) and the rotating plate at the bottom. b The multi-launcher from the bottom showing the rotating plate and a projectile (marked by an arrow) in its individual launcher through the plate's drop hole 
Table 1 Projectiles and target materials of the three COLLIDE and the PRIME-3 campaigns

\begin{tabular}{|c|c|c|c|c|c|c|c|c|}
\hline \multirow[t]{2}{*}{ COLLIDE campaign } & \multirow[t]{2}{*}{ IBS } & \multicolumn{4}{|l|}{ Projectile } & \multirow[b]{2}{*}{ Target material } & \multirow[b]{2}{*}{ Outcome } & \multirow[b]{2}{*}{ Coefficient of restitution } \\
\hline & & Diameter $[\mathrm{cm}]$ & Mass [g] & Material & Speed $[\mathrm{cm} / \mathrm{s}]$ & & & \\
\hline 1 & 1 & 0.96 & 0.99 & Teflon & 14.80 & JSC-1 & $\mathrm{R}$ & 0.028 \\
\hline 1 & 4 & 0.96 & 0.99 & Teflon & 17.10 & JSC-1 & $\mathrm{R}$ & 0.022 \\
\hline 1 & 6 & 0.96 & 0.99 & Teflon & 90.00 & JSC-1 & $\mathrm{R}$ & 0.03 \\
\hline 2 & 1 & 2.00 & 10.71 & Quartz & 110.00 & Quartz sand & E & 0.01 \\
\hline 2 & 2 & 2.00 & 9.64 & Quartz & 3.60 & Quartz sand & E & N/A \\
\hline 2 & 3 & 2.00 & 10.29 & Quartz & 1.29 & Quartz sand & E & N/A \\
\hline 2 & 4 & 2.00 & 8.66 & Quartz & 81.00 & Quartz sand & E & 0.015 \\
\hline 2 & 5 & 2.00 & 8.98 & Quartz & 12.20 & JSC-1 & E & N/A \\
\hline 2 & 6 & 2.00 & 10.62 & Quartz & 28.00 & Quartz sand & E & 0.02 \\
\hline 3 & 2 & 2.05 & 10.55 & Quartz & 26.5 & Quartz sand & R & $9.3 \times 10^{-3}$ \\
\hline 3 & 3 & 2.05 & 10.55 & Quartz & 29.3 & JSC Mars-1 & E & N/A \\
\hline PRIME-3 flight & Box & & & & & & & \\
\hline 1 & 1 & 2.05 & 9.82 & Quartz & 48.45 & Quartz sand & E & N/A \\
\hline 1 & 3 & 2.05 & 11.76 & Quartz & 49.72 & Quartz sand & E & N/A \\
\hline 1 & 4 & 2.05 & 30.75 & Brass & 43.19 & Quartz and & A & N/A \\
\hline 1 & 5 & 2.05 & 9.9 & Quartz & 43.75 & Quartz and & E & N/A \\
\hline 1 & 6 & 2.05 & 28.2 & Brass & 19.87 & Quartz sand & $x$ & N/A \\
\hline 1 & 7 & 2.05 & 9.82 & Quartz & 33.32 & JSC-1 & $\mathrm{R}$ & 0.078 \\
\hline 1 & 8 & 2.05 & 28.2 & Brass & 19.56 & JSC-1 & $x$ & N/A \\
\hline 2 & 2 & 2.05 & 28.2 & Brass & 16.21 & Quartz sand & $\mathrm{R}$ & 0.046 \\
\hline 2 & 3 & 2.05 & 11.76 & Quartz & 31.52 & Quartz sand & A & N/A \\
\hline 2 & 4 & 2.05 & 30.75 & Brass & 38.4 & Quartz sand & A & N/A \\
\hline 2 & 5 & 2.05 & 9.9 & Quartz & 38.73 & Quartz sand & E & 0.025 \\
\hline 2 & 6 & 2.05 & 28.2 & Brass & 13.6 & Quartz sand & $x$ & N/A \\
\hline 2 & 7 & 2.05 & 9.82 & Quartz & 5.55 & JSC-1 & R & 0.276 \\
\hline 2 & 8 & 2.05 & 28.2 & Brass & 14.45 & JSC-1 & $\mathrm{R}$ & 0.091 \\
\hline 3 & 1 & 2.05 & 9.82 & Quartz & 24.75 & Quartz sand & E & N/A \\
\hline 3 & 3 & 2.05 & 11.76 & Quartz & 26.38 & Quartz sand & E & N/A \\
\hline 3 & 4 & 2.05 & 30.75 & Brass & 31.84 & Quartz sand & A & N/A \\
\hline 3 & 5 & 2.05 & 9.9 & Quartz & 48.18 & Quartz sand & A & N/A \\
\hline 3 & 6 & 2.05 & 28.2 & Brass & 15.2 & Quartz sand & $x$ & N/A \\
\hline 3 & 8 & 2.05 & 28.2 & Brass & 4.02 & JSC-1 & R & 0.229 \\
\hline 4 & 1 & 2.05 & 9.82 & Quartz & 25.85 & JSC-1 & $E$ & 0.075 \\
\hline 4 & 3 & 2.05 & 11.76 & Quartz & 20.94 & Quartz sand & E & 0.020 \\
\hline 4 & 4 & 2.05 & 30.75 & Brass & 27.77 & $J S C-1$ & A & N/A \\
\hline 4 & 5 & 2.05 & 9.9 & Quartz & 52.93 & Quartz sand & A & N/A \\
\hline 4 & 6 & 2.05 & 28.2 & Brass & 10.02 & Quartz sand & $x$ & N/A \\
\hline 4 & 8 & 2.05 & 28.2 & Brass & 13.38 & JSC-1 & $\mathrm{R}$ & 0.055 \\
\hline
\end{tabular}

The table of the 108 PRIME-1 campaign impacts can be found in Colwell et al. (2008). No coefficients of restitution could be measured for the PRIME-1 impacts. $R$ rebound with no ejecta, E ejecta production, A asteroid-level parabola $(0.05 \mathrm{~g}), X$ no data available (regolith pillar), N/A no rebound from the projectile on the target could be observed 


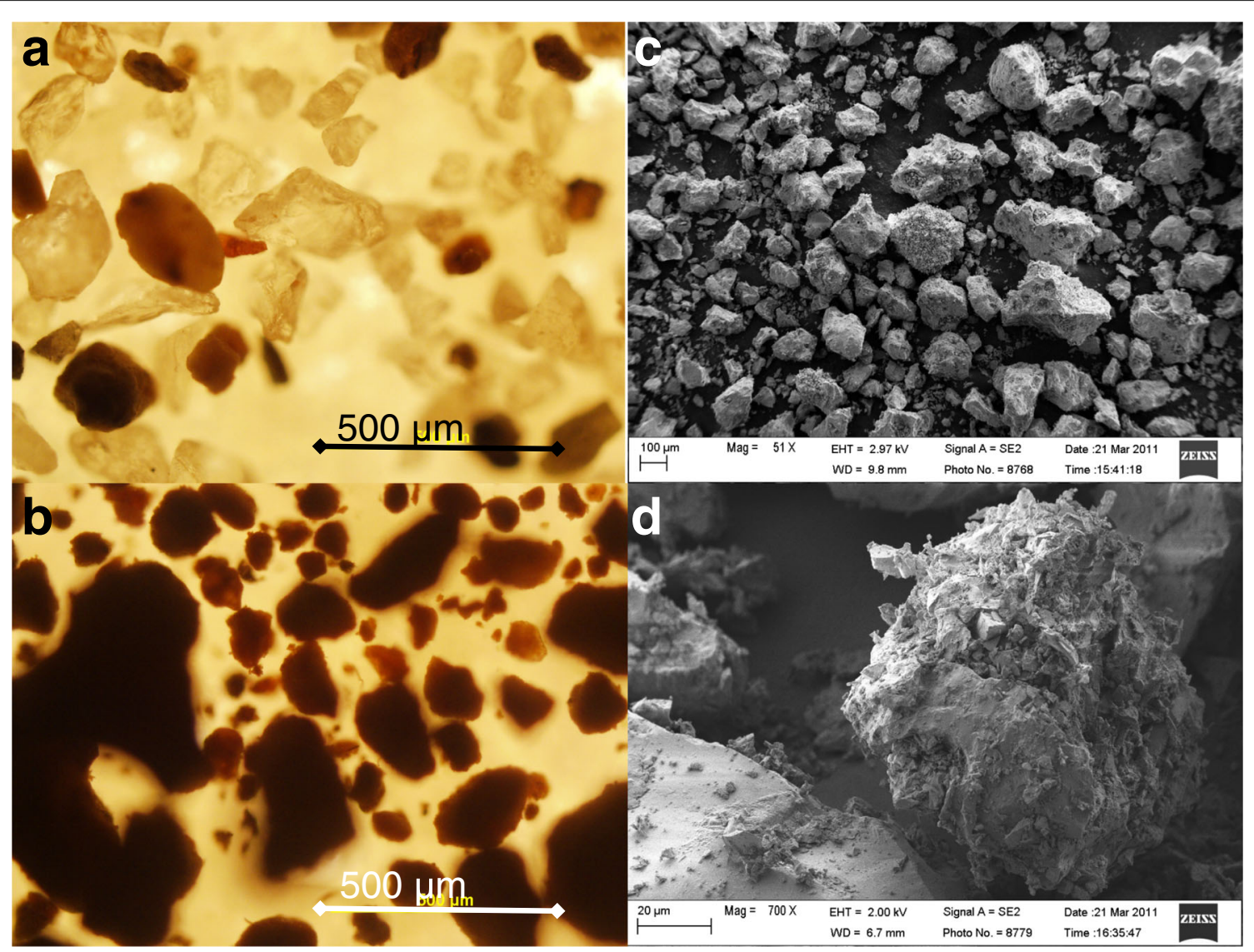

Fig. 3 Optical and scanning electron microscope (SEM) pictures of the simulants used in COLLIDE and PRIME experiments. a Quartz sand and $\mathbf{b}$ JSC Mars-1 and $\mathbf{c ~ J S C - 1 ~ l u n a r ~ s i m u l a n t s . ~ T h e ~ s a m p l e s ~ w e r e ~ p r e p a r e d ~ i n ~ t h e ~ s a m e ~ m a n n e r ~ a s ~ f o r ~ t h e ~ e x p e r i m e n t s , ~ b y ~ s i e v i n g ~ t h e ~ d i f f e r e n t ~ g r a i n s ~ t o ~ b e l o w ~}$ $250 \mu \mathrm{m}$. d shows the details of the surface texture of JSC-1 grains

deep ( $>>250 \mu \mathrm{m}$ grain diameter), we expect that the tray had no influence on the target response to the impacts performed in COLLIDE and PRIME.

The trays were consistently filled using the same procedure, in order to reproduce the same target density. This filling procedure consisted in the pouring of simulant material into the tray using a funnel, followed by the leveling of the surface using a flat metal ruler. We measured the porosities of targets produced in this manner in the laboratory (30 times) by filling the tray using this procedure, then measuring the mass of the target material using a scale. Knowing the container volume and the bulk density of the grains, we determined target porosities consistently ranging from 0.4 to 0.5 . While the compaction level of fine grains at the surface of asteroids is not well determined, the bulk porosity of "rubble-pile" asteroids was estimated to be of this order by Britt et al. (2002), indicating that such porosity levels are relevant for the study of low-velocity impacts on asteroid surfaces.
In the COLLIDE experiment setup, the target was contained by a sliding door, with no particular measures to prevent further compaction through post-preparation handling and launch vibrations. However, the COLLIDE3 videos, which have a high enough resolution to show details of the target tray filling after the door opened during the rocket flight, show no volume reduction of the target sample. The target compaction during COLLIDE- 1 and COLLIDE- 2 could not be quantified due to the low resolution of the video data, but the projectile impact location indicates that the target surface was not significantly shifted from its original position, so that we do not estimate porosities much below 0.4 during the experiment runs.

\section{PRIME}

Flight campaigns PRIME flew on three experiment campaigns on the NASA KC-135 and C-9 parabolic airplanes. The first flight campaign induced five flight weeks between July 2002 and May 2003 and is described in 
Colwell et al. (2008). The PRIME-1 campaign produced 108 successful impact recordings.

From the results of this first campaign, it was clear that the regolith was very sensitive to the residual accelerations of the aircraft. Therefore, the PRIME-2 hardware was modified to be partially free-floating. In this setup, the individual impact boxes could be pulled out of the overall payload structure, which was bolted to the airplane floor. The boxes were still attached to the structure by an electrical umbilical. Unexpectedly, the residual accelerations of the plane during parabolas were still high enough to introduce torques onto the impact boxes via this umbilical, generating a worse acceleration environment than during PRIME-1, and no scientific data could be collected.

The PRIME- 3 hardware was designed to be entirely free-floating. Each impact box had its own electronics attached to it, including a battery pack for power. The following paragraph describes the hardware flown during the PRIME-3 1-week flight campaign in August 2014. The PRIME-3 campaign produced 25 impact recordings. During the flight campaign, the pilots flew a number of parabolas at $0.05 g$ (named "asteroid-level" parabolas by the NASA flight support team) in addition to the usual microgravity ones. Out of the 25 recorded impacts, 7 were at asteroid level. Details on the PRIME-3 campaign can be found in Colwell et al. (2016).

Hardware Figure 4 shows the PRIME-3 hardware. It is composed of eight individual impact boxes that are kept in a structure bolted to the airplane floor. Each box can be slid out of the structure and used to perform an experiment run while completely free-floating during a parabola. The impact boxes were inherited from PRIME1 and PRIME- 2 and modified to allow for an autonomous experiment performance: while the regolith tray and launch mechanisms are similar, the electronics were re-designed to actuate them externally using a wireless signal. One camera was used to record the impacts in the individual chambers as it was attached to a bracket that could be switched from box to box. The camera used was also a GoPro Hero 3+ black edition. More details on the inside of the impact boxes can be found in Colwell et al. (2008).

During asteroid-level parabolas, the impactor box was placed on the floor of the airplane and the projectile launch initiated at the beginning of the parabola.

Projectiles and simulants The list of projectiles and simulants for the 108 impacts recorded during the PRIME-1 campaign can be found in Colwell et al. (2008). Table 1 summarizes the projectiles and simulants used during the PRIME-3 campaign.

The target materials and preparation methods were the same as for COLLIDE (see "Projectiles and simulants" section for details). The only difference with the COLLIDE targets is an additional contraption on the tray door that slightly compresses the target after preparation and through the pre-flight handling process. As the PRIME tray doors open by rotation rather than sliding, a plate
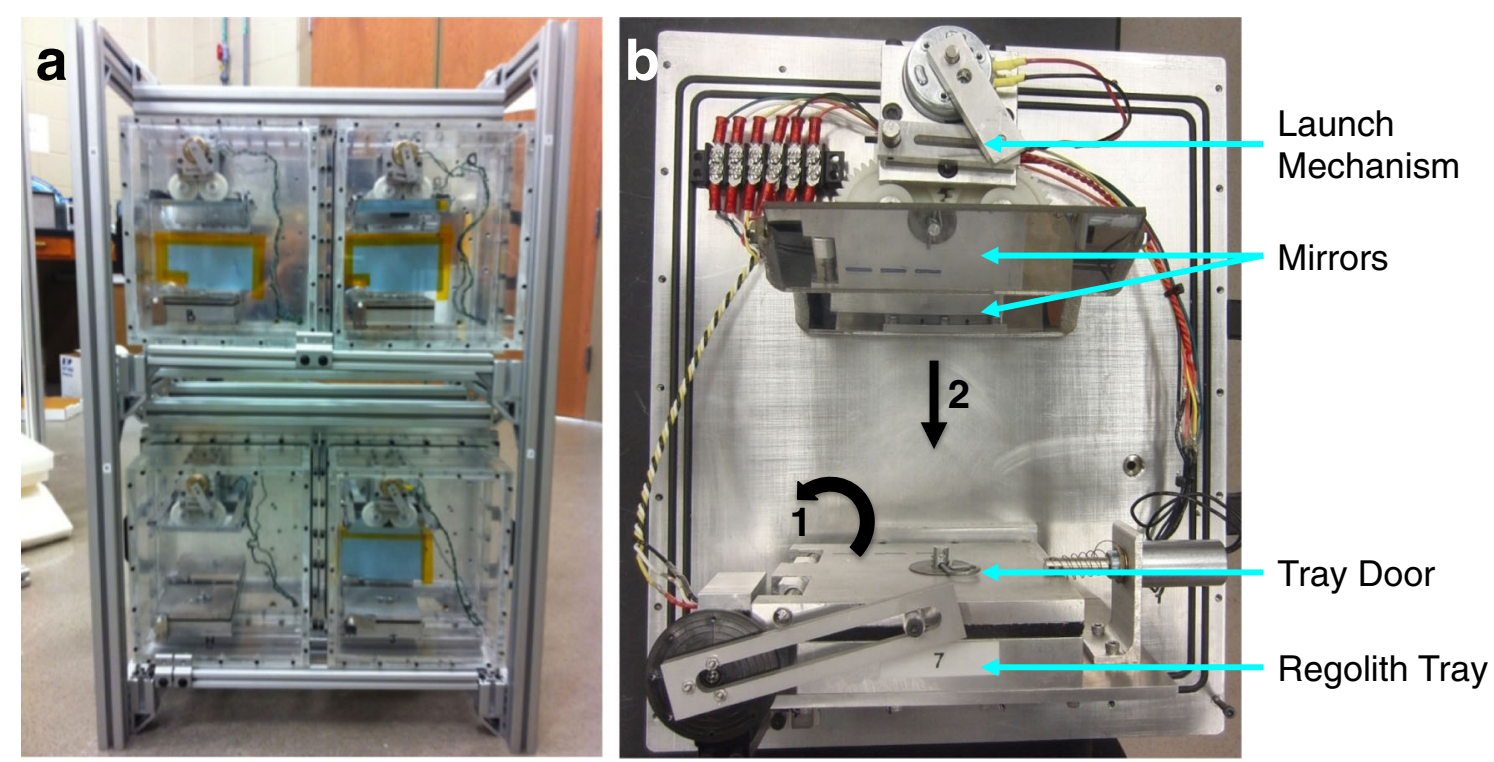

Fig. 4 PRIME-3 hardware. a The payload structure that is bolted to the aircraft floor and holds the eight impact boxes (only four are visible in this front view). $\mathbf{b}$ Interior of an impact box showing the regolith tray and the launch mechanism. During an experiment run, the tray door rotates to open (1) before the projectile gets launched towards the regolith bed (2) 
was attached on the inner side facing the sample. Using four weak springs, this plate applies pressure on the target sample when the tray door is closed. In this way, the sample is jammed and its porosity remains unchanged (0.4 to 0.5 as described in "Projectiles and simulants" section) during loading of the experiment boxes onto the aircraft.

\section{Results}

As the target size distribution and porosity were the same for all the COLLIDE and PRIME campaigns, we combined the collected data sets. This section presents how we analyzed the impacts and the results we obtained, integrating all the data collected.

The platforms used to collect data in microgravity induced residual accelerations on the experiment hardware attached to them. These accelerations were around $10^{-3} g$ in the Space Shuttle (Colwell and Taylor 1999) and $10^{-2} g$ on the parabolic aircraft (Colwell
2003) and on Blue Origin's New Shepard rocket (Wagner and DeForest 2016). The free-floating experiment boxes of PRIME-3 offered the best microgravity quality: with only air drag acting on the box moving at very low speeds $(<1 \mathrm{~mm} / \mathrm{s})$, no residual acceleration could be detected from our video data. This implies that these experiment runs were performed at residual accelerations under our detection threshold, $10^{-4} g$. In addition to these microgravity experiment runs, the PRIME-3 flights also allowed us to perform seven experiment runs at $0.05 g$.

Across all the data collected, four types of collision outcome were observed:

- Collisions producing ejecta with an embedded projectile (squares in Fig. 5): in these collisions, no motion of the projectile after the impact is detected. Either the ejecta blanket or individual particles could be tracked and their speed determined.

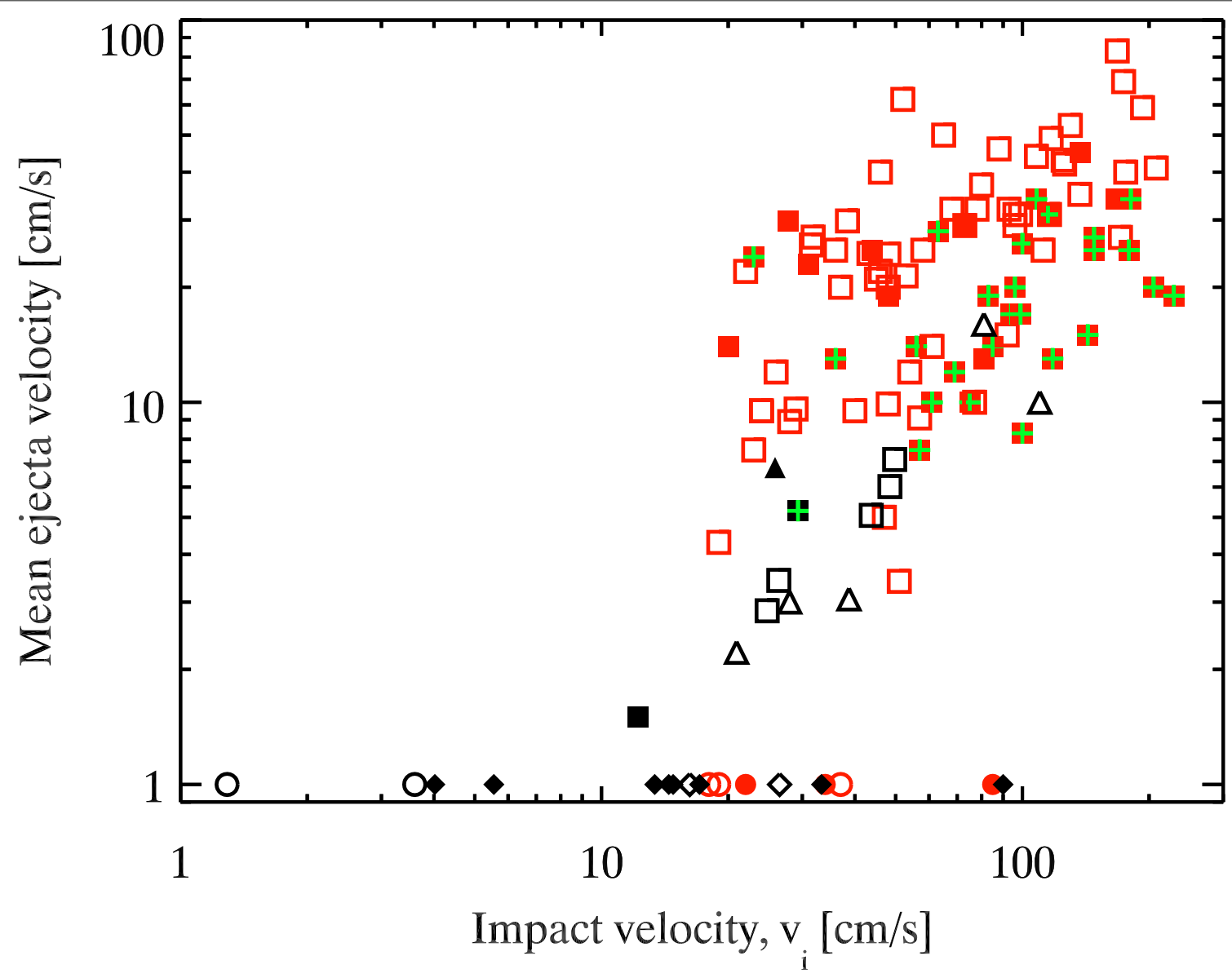

Fig. 5 Outcomes of all the low-velocity impacts observed in PRIME-1, PRIME-3, and the COLLIDE campaigns as a function of the impact velocity. Circles: projectile embedded, no ejecta; diamonds: projectile rebound, no ejecta; squares: projectile embedded, ejecta production; triangles: projectile rebound, ejecta production; open symbols: quartz sand target; filled symbols: JSC-1 target; green plus sign: JSC Mars-1 target; red: reduced gravity $\left(\sim 10^{-2} \mathrm{~g}\right)$; black: microgravity $\left(<10^{-4} \mathrm{~g}\right)$. All impacts producing no ejecta are shown at a mean ejecta velocity of $1 \mathrm{~cm} / \mathrm{s}$ for the clarity of the graph, even though no speed was measured as no ejecta was observed 
- Collisions producing ejecta with projectile rebound (triangles in Fig. 5): the projectile can be tracked after the collision. In addition to the ejecta speeds, a coefficient of restitution could be measured for the projectile.

- Collisions with no ejecta but projectile rebound (diamonds in Fig. 5). The coefficient of restitution for the projectile could be measured.

- Collisions with no ejecta and embedded projectile (circles in Fig. 5).

Figure 5 shows these outcomes as a function of the impact velocity. In this graph, all impacts not producing any ejecta were placed at a mean ejecta velocity of $1 \mathrm{~cm} / \mathrm{s}$. This is for the purpose of representing all the data on the same graph only, as no speed could be measured on absent ejecta (and a representation at 0 is not possible on a logarithmic scale). The following paragraphs give some additional details on these results for quartz sand and JSC-1 target materials.

JSC Mars-1 behaved similarly to quartz sand in reduced gravity (the JSC Mars-1 data points seen in Fig. 5 are mostly from the PRIME- 1 campaign; see Table 1 in Colwell et al. (2008)). This can be explained by the similar grain shape between these two simulants (Fig. 3a, b). In microgravity, only one impact was available for data analysis, which was not enough for analyzing this set of data.

\section{Ejecta production}

Impacts in COLLIDE-2, COLLIDE-3, PRIME-1, and PRIME-3 resulted in the production of an ejecta blanket. In the PRIME-1 and most of the COLLIDE- 2 data (Colwell 2003), individual ejecta particles could not be distinguished due to the low camera resolution. However, features of the ejecta blanket, in particular the fastest particles forming the upper edge of the blanket (named "corner" in Colwell et al. (2008)), were identified and allowed for the measurement of the upper end of the ejecta velocity distribution.

In PRIME-3, the camera resolution allowed for the individual tracking of ejecta particles. For these experiments, we were able to measure a mean ejecta velocity by directly tracking 30 particles for each impact, using the Spotlight software developed at NASA Glenn Research Center (Klimek and Wright 2006). However, due to the nature of the video data collected, the tracking of individual particles is limited to optically thin portions of the ejecta blanket. This includes but is not restricted to the ejecta "corner" mentioned above. Particle tracking was performed by three people independently in order to reduce the measurement errors. The ejecta grain velocity distribution obtained for each impact was normal and could be fit by a Gaussian curve. Details on the tracking methods can be found in Colwell et al. (2016) (see their Figs. 5 and 6). For PRIME-3 and COLLIDE-3, the characteristic ejecta velocity was chosen to be the Gaussian mean of velocity distribution of the tracked particles.

Figure 6 shows the results of the ejecta speed measurements for PRIME-1 and PRIME-3. The 1- $\sigma$ error bars for the Gaussian mean determination are shown for the PRIME-3 data points. COLLIDE-3 produced ejecta for only one impact, and the target material was JSC Mars-1. As stated above, JSC Mars-1 behaved similarly to quartz sand, and we are not including JSC Mars-1 results here. The plateau formed by the PRIME-1 microgravity data points (black asterisks representing quartz sand impacts) around $10 \mathrm{~cm} / \mathrm{s}$ for impact energies below $10^{4}$ ergs is due to the residual accelerations induced by the parabolic aircraft on the experiment boxes (on the order of $10^{-2} \mathrm{~g}$ ). Only particles ejected with energies able to overcome the effect of this ambient gravity field were lifted from the target. For this reason, the slowest moving ejecta has a minimum speed, set by this $g$-level, and the measured velocities follow a plateau. This plateau also formed in PRIME-3 microgravity data, but at ejecta velocities around a few centimeters per second, indicating that residual accelerations were successfully reduced by the free-floating hardware configuration, compared to PRIME-1, which was attached to the aircraft frame.

The scatter of the PRIME-1 data points towards higher ejecta velocities was induced by the observational bias produced by the tracking method used: tracking the ejecta "corner" described above limits the recorded speeds to the fastest moving particles and is therefore only representative of the upper end of the ejecta velocity distribution: there is therefore a tendency to measure higher ejecta velocities at the same impact energies. However, the data points show a trend followed by the lower limit of the measured velocities: to guide the eye, we fit a power law to the impacts into quartz sand in microgravity. The index obtained is 1.35 . For all impacts into JSC-1, a fitted power law index is at 0.50 . We collected only one data point for JSC-1 ejecta at microgravity levels, which lies below this power law fit. Further data collection on JSC-1 ejectaproducing impacts in microgravity will be required in order to evaluate if this point is indicative of a different ejecta behavior between the two gravity levels.

The ejecta speeds measured for impacts at $0.05 \mathrm{~g}$ during the PRIME- 3 campaign are of the same range as the ones measured during the PRIME-1 campaign, where microgravity parabolas were flown, but the experiment hardware was fixed to the aircraft frame. This demonstrates that the residual accelerations produced on the experiment when attached to the aircraft are comparable to $10^{-2} \mathrm{~g}$ and that our method for determining the average ejecta speeds provides similar values than the method used in Colwell et al. (2008). 


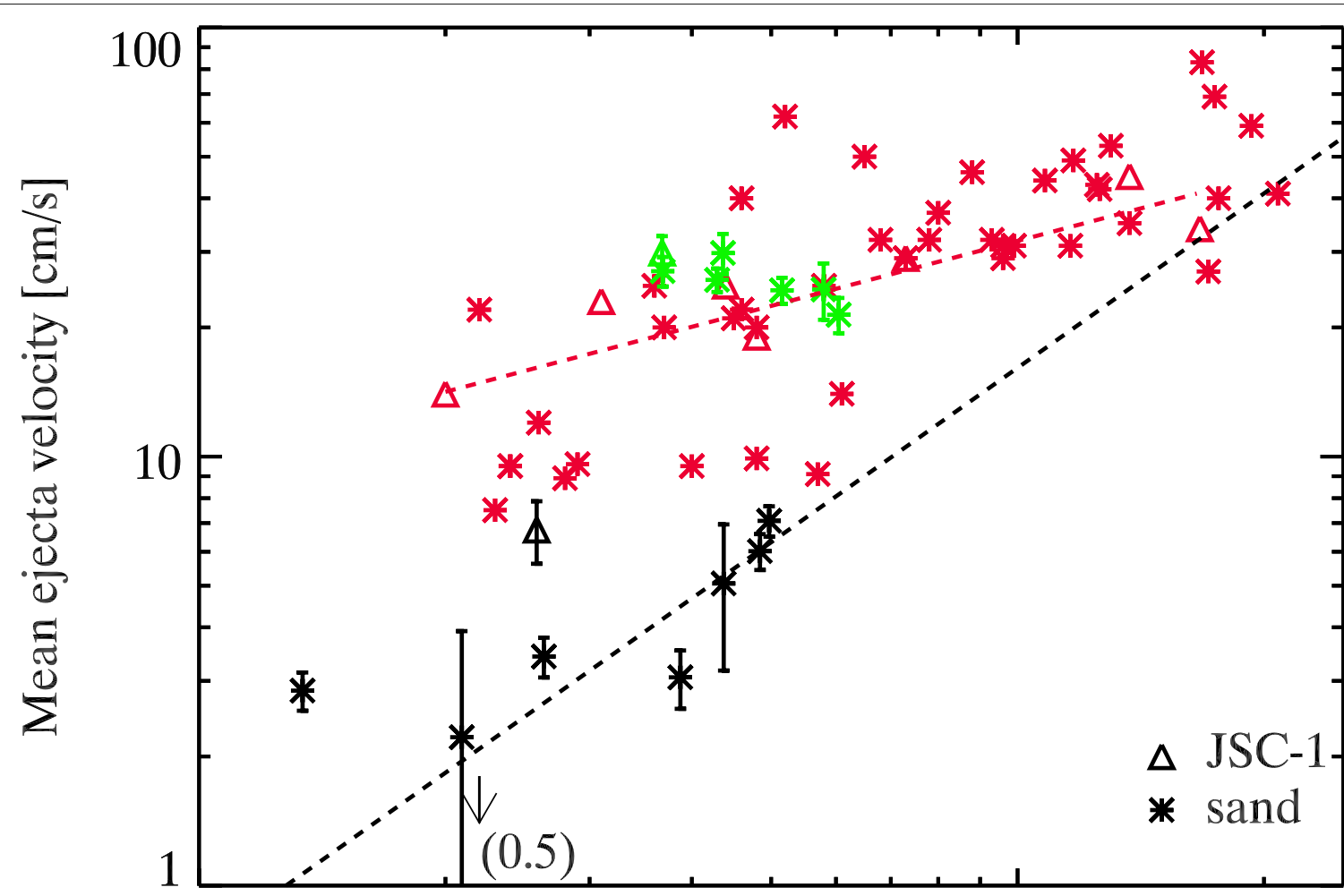

10

100

\section{Impact velocity $[\mathrm{cm} / \mathrm{s}]$}

Fig. 6 Combined PRIME-1 and PRIME-3 ejecta velocity measurements as a function of the impact velocity. PRIME-1 data is shown by red symbols. For PRIME-3, black symbols show impacts in the free-floating box $\left(<10^{-4} \mathrm{~g}\right)$, while green symbols show impacts at $0.05 \mathrm{~g}$. Impacts into quartz sand are marked by asterisks, and impacts into JSC-1 by triangles. Error bars are not shown for PRIME-1: they were smaller than the symbol size (Colwell 2003). PRIME-3 error bars represent the 1- $\sigma$ error of the Gaussian fit to the velocity distribution of tracked particles. The dashed black line is a power law fit to the microgravity impacts in sand (index 0.50), and the red dashed line is a power law fit to all the impacts into JSC-1 (index 1.35)

We defined the effective coefficient of restitution of the impacts as the ratio between the average ejecta velocity and the projectile impact velocity and investigated its relationship to the impact velocity. These effective coefficients of restitution showed no correlation and a uniform distribution over the available range of impact velocities (Fig. 7). We calculated the overall average effective coefficient of restitution to be $0.39 \pm 0.15$. This value is about twice the one derived by Deboeuf et al. (2009) for impacts of centimeter-sized spheres into $100 \mu \mathrm{m}$ glass bead beds. Deviations to our mean value were much higher than for the measurements performed in Deboeuf et al. (2009). When separating the impacts into quartz sand from the ones into JSC-1, we obtained average effective coefficients of restitution of $0.38 \pm 0.15$ and $0.45 \pm 0.16$, respectively. When separating between impacts in low gravity and microgravity, we obtained values of $0.43 \pm 0.14$ and $0.15 \pm 0.04$, respectively.

\section{Projectile rebound}

A number of impacts during the COLLIDE-1, COLLIDE-3, and PRIME-3 campaigns showed a rebound of the projectile after impact on the target and allowed for the measurement of a coefficient of restitution $\epsilon=\frac{v_{f}}{v_{i}}$, $v_{i}$ and $v_{f}$ being the projectile velocities before (initial) and after (final) the impact, respectively (see Table 1). Figure 8 shows the measured coefficients of restitution as a function of the impact energy. For speeds above $30 \mathrm{~cm} / \mathrm{s}$, impacts systematically produced an ejecta blanket and coefficients of restitution of the projectile could either not be measured or were of the order of $10^{-2}$.

At impact speeds between about 20 and $30 \mathrm{~cm} / \mathrm{s}$, both ejecta production and projectile rebound without ejecta were observed. Below $20 \mathrm{~cm} / \mathrm{s}$, only rebounds without ejecta were observed. In order to compare our results to the experimental work of Katsuragi and Blum (2017), we fit a power law to this set of data. In Katsuragi and 


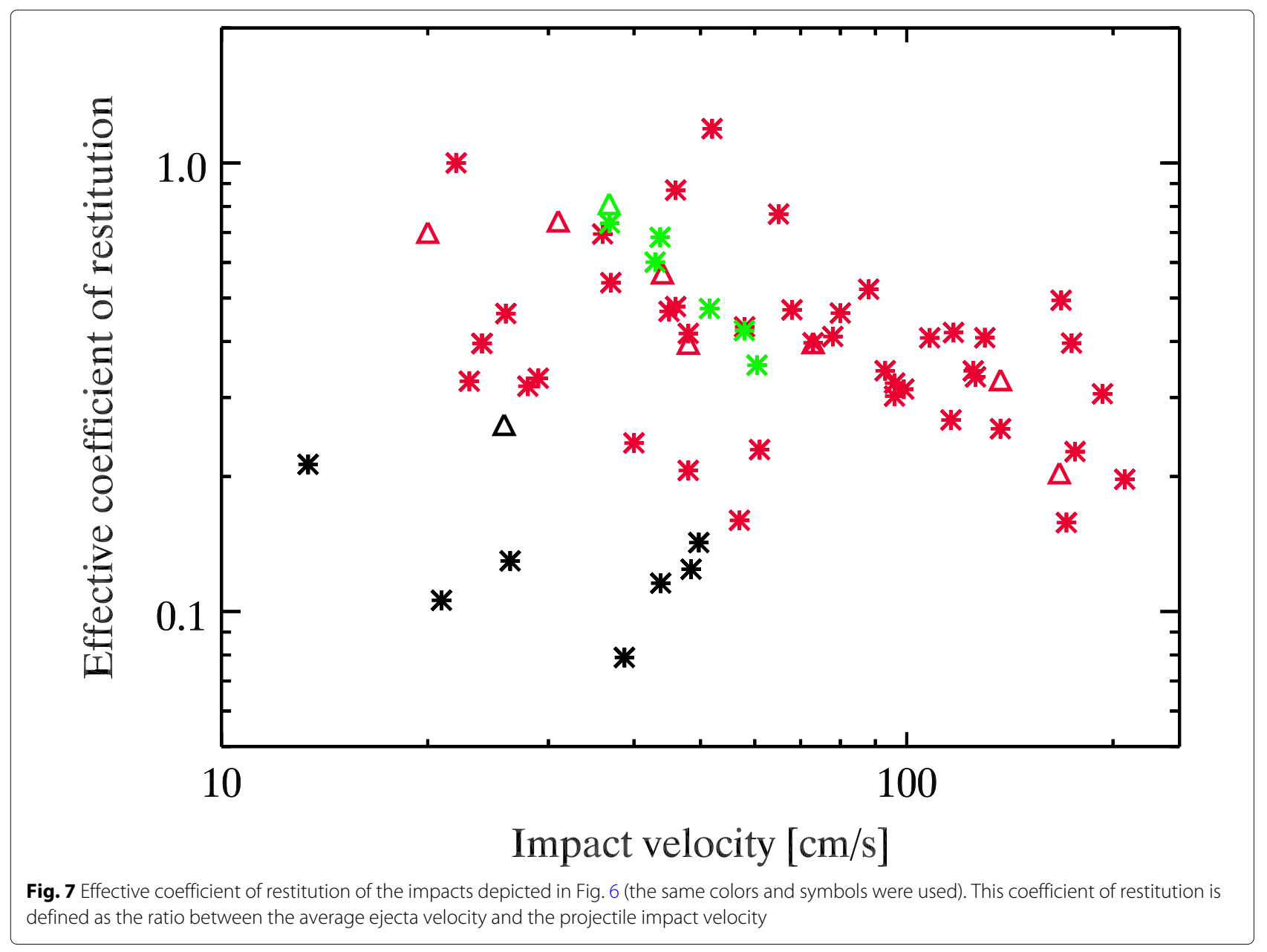

Blum (2017), spherical projectiles of about $1 \mathrm{~cm}$ in diameter are dropped into aggregates composed of $750 \mu \mathrm{m}$ $\mathrm{SiO}_{2}$ monomer grains with a packing density of $\Phi=0.35$. The index we obtain with our data is -0.27 , similar to the value of $-1 / 4$ found by Katsuragi and Blum (2017). We note, however, that if we separate the data sets from COLLIDE and PRIME, we obtain indexes of -0.10 and -0.50 , respectively, a difference mostly due to the two very low-energy impacts into JSC-1 of the COLLIDE1 flight. As mentioned in Katsuragi and Blum (2017), this overall index value is twice as high as expected from the theory of an elastic sphere impacting a plane surface (Johnson 1985; Thornton and Ning 1998). This indicates that energy absorption in a bed of granular material is not entirely captured by the mechanics of elastic surfaces.

\section{Projectile penetration depth}

In the PRIME-3 video data, the resolution is high enough to determine the maximum penetration depth of the projectile into the target for 21 impacts. This maximum penetration depth is defined as the distance between the bottom of the projectile and the target surface. Figure 9 shows these results as a function of the equivalent total drop distance $H$. This distance was derived to be able to compare our results with the ones obtained by Katsuragi and Blum (2017): $H=h+z_{\max }$, where $h$ is the drop height (experiments in Katsuragi and Blum (2017) are performed in $1 g$, and the drop height is used to control the impact velocity), and $z_{\max }$ is the maximum penetration depth of the projectile into the target. As the impact velocity was not determined by the drop height in PRIME experiments, but by the stored potential energy in the spring of the projectile launcher, we calculate an equivalent $h$ from the impact velocity $v_{i}$ by equating the $1 g$ potential energy with our kinetic impact energy as follows:

$$
\begin{aligned}
E_{1 g} & =E_{0 g} \\
m g h & =\frac{1}{2} m v_{i}^{2} \\
h & =\frac{v_{i}^{2}}{2 g}
\end{aligned}
$$




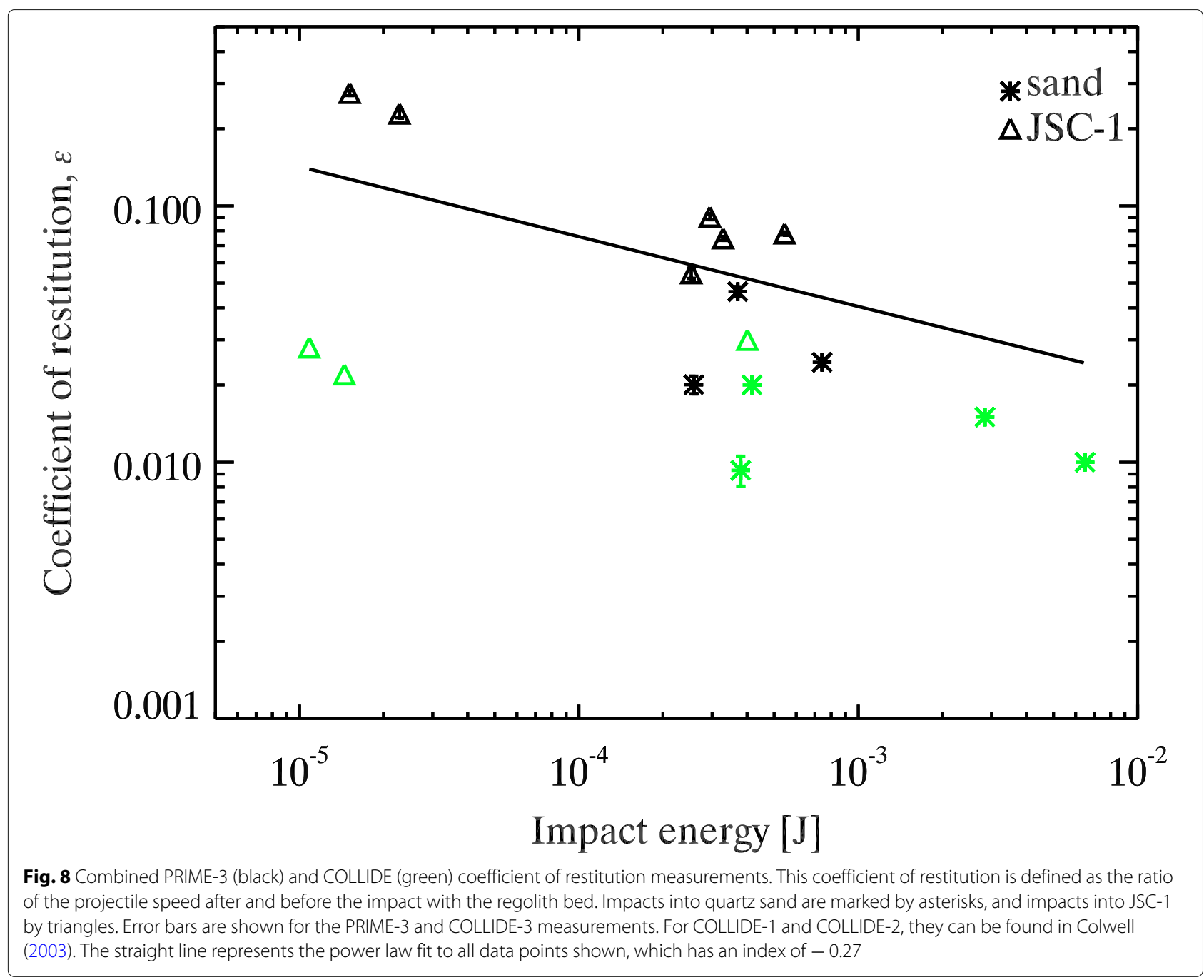

with $g=9.81 \mathrm{~m} / \mathrm{s}$. As shown by the index 1 power law in Fig. 9, $H$ is dominated by $z_{\max }$ in half of the impacts. This is due to the very low impact velocities, inducing equivalent drop heights $h \ll z_{\max }$, so that $H \sim z_{\max }$. We do not observe any correlation between the maximum penetration depth and the computed total equivalent drop distance. This is in contrast with the relation $z_{\max } \propto H^{1 / 3}$ observed in Katsuragi and Blum (2017), representative of a scaling by impact energy. A scaling by momentum of the form $z_{\max }=A m_{p}^{\alpha} v_{i}^{\beta}$, with $m_{p}$ and $v_{i}$ being the projectile mass and impact velocity, respectively, and $A, \alpha$, and $\beta$ fit parameters, as performed in Güttler et al. (2009), was not successful either.

\section{Grain behavior with decreasing gravity level}

From the experimental results presented here, we observed differences in the response of the target to lowvelocity impacts in reduced gravity $\left(\sim 10^{-2} g\right)$ and microgravity $\left(<10^{-4} g\right)$. Figure 5 shows that compared to the total number of impacts in reduced gravity $\left(\sim 10^{-2} \mathrm{~g}\right)$, only a few of them did not produce ejecta: the vast majority of impacts generated an ejecta curtain. In addition, none of the reduced-gravity impacts lead to a rebound of the projectile (no red triangles or diamonds). In particular, when no ejecta was produced around the lowest impact speeds observed $(\sim 20 \mathrm{~cm} / \mathrm{s})$, only projectile embedding in the target could be seen. For the same impact speeds, a microgravity environment $\left(<10^{-4} \mathrm{~g}\right)$ lead to the rebound of the projectile. In fact, embedding into the target was only seen in the two impacts under $4 \mathrm{~cm} / \mathrm{s}$. All other impacts than those without ejecta showed a rebound of the projectile.

Projectile rebound was also observed in combination with an ejecta curtain in half of the impacts observed in microgravity (black triangles). Even though Fig. 8 shows that the coefficient of restitution of these rebounds is much smaller compared to the ones with no ejecta production (diamonds), this behavior demonstrates the 


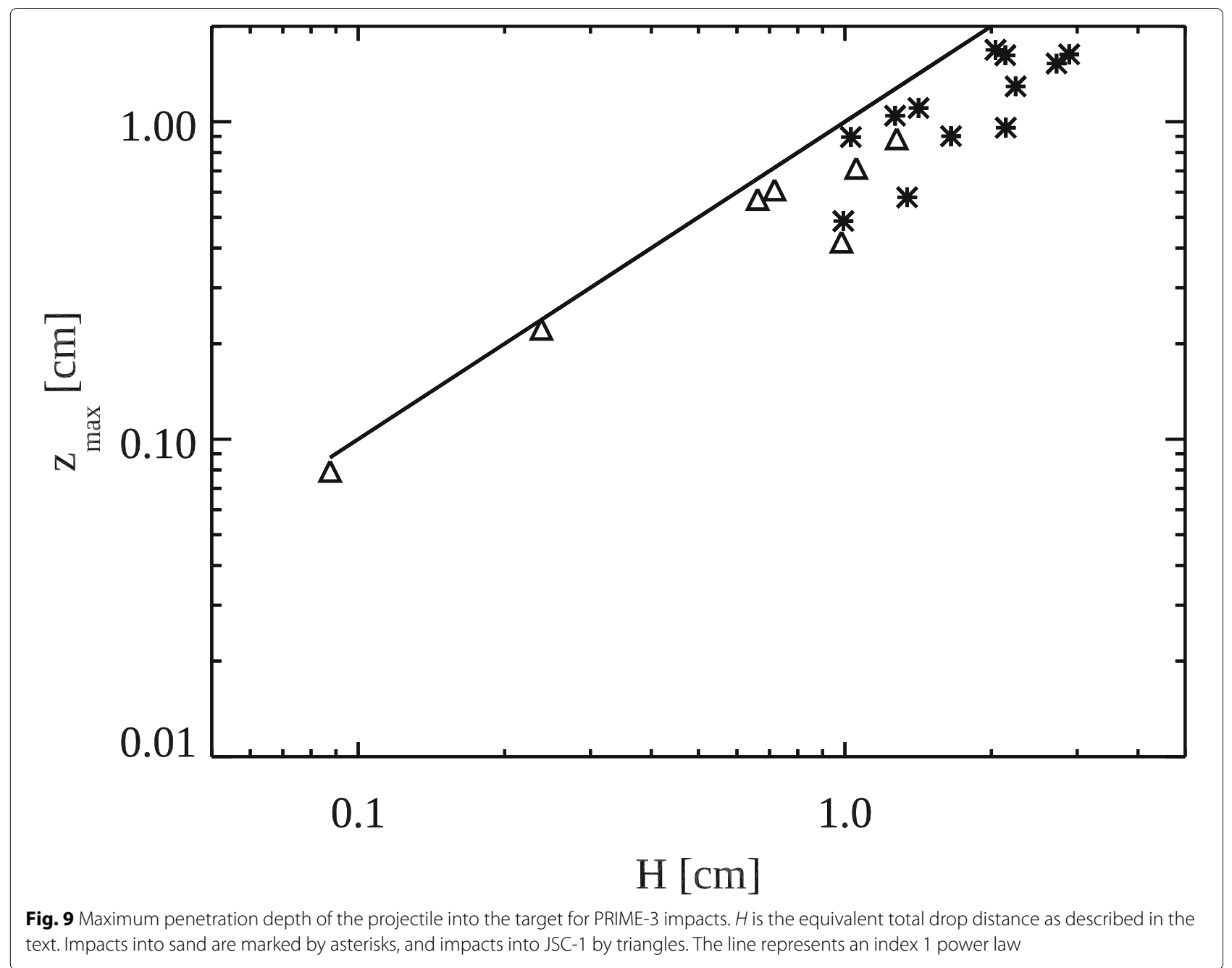

differences in target response in reduced- and microgravity environments.

It can also be noted that for the same impact speed, ejecta is faster on average in reduced gravity compared to microgravity (red symbols are above black symbols in Fig. 5). In addition, less mass is ejected than in microgravity. Figure 10 shows the average ejecta speeds for four levels of ejected mass: as the ejecta mass cannot be measured after each experiment run (the return to $1 g$ mixes the target material from the tray and the ejected particles), an estimation was performed from the video images. Four levels of ejecta mass were identified: no ejecta (0), individual particles detaching from the target (1), ejected mass of the order of the projectile mass (2), and ejected mass much higher than the projectile mass (3) (we followed the same numbering as in Colwell (2003)). In Fig. 10, the red symbols show reduced-gravity impacts $\left(\sim 10^{-2} g\right)$, and black symbols microgravity impacts $\left(<10^{-4} g\right)$. The impact velocity given for each target type and ejecta mass level is the average of all impacts that created the same amount of ejecta. From the sand targets, we can see that the same average impact speed results in higher ejecta masses in microgravity compared to reduced gravity. Together with Fig. 5, this shows that fewer target particles are ejected in reduced gravity, but their ejection speed is higher.

Figure 10 also shows that no ejecta blanket was observed in microgravity with JSC- 1 targets. While this is also due to the fact that only very few impacts were performed in JSC-1 in microgravity at speeds $>50 \mathrm{~cm} / \mathrm{s}$, the nature of the target plays a role in the ejecta mass produced: compared to quartz sand particles, which are rounded and considered cohesionless in vacuum (once the air humidity is removed), JSC-1 particles are more angular and behave like a cohesive powder. Figure 5 shows that only two out of eight impacts into JSC-1 at $>10 \mathrm{~cm} / \mathrm{s}$ resulted in ejecta production, and these were only individual particles detaching instead of an ejecta blanket. As we can see in Fig. 10, the impact speed does not seem to influence the production if these individual particles in JSC-1 targets, 


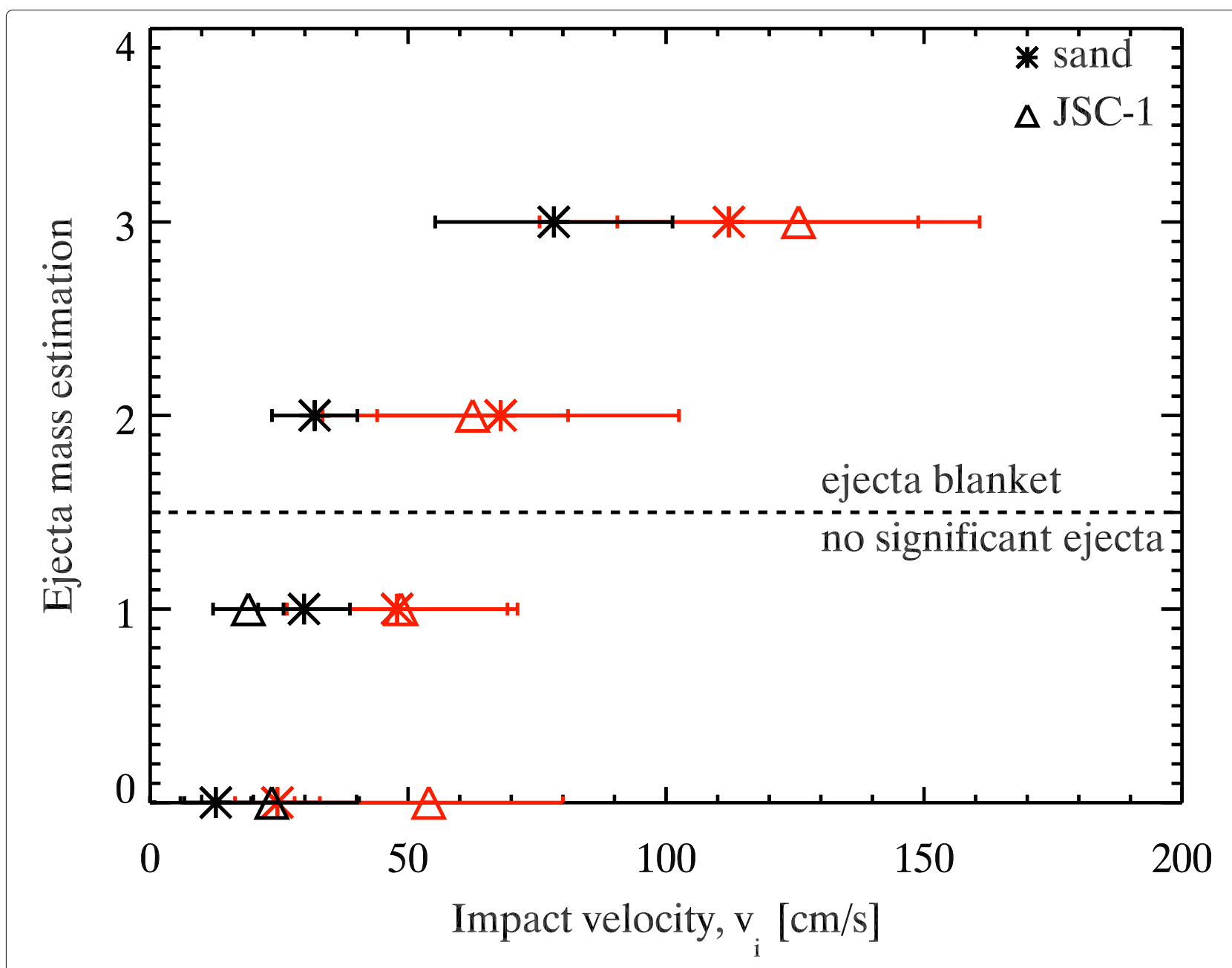

Fig. 10 Arbitrary quantified amount of ejecta for all collected data on sand and JSC-1 targets. The amount of ejecta mass was quantified as follows: $0=$ no ejecta; 1 = ejection of single particles; 2 = ejecta blanket, mass of the order of the projectile mass; and $3=$ ejecta blanket, mass much higher than the projectile mass. The impact velocity given for each target type is the average of all impacts that created the same amount of ejecta. Asterisk = quartz sand target, triangle $=$ JSC-1 lunar simulant target, black = microgravity impacts, red = reduced-gravity impacts. The dotted line marks the limit between no significant ejecta produced (either none at all or only individual particles detaching) and an ejecta blanket

compared to quartz sand targets, which display a more consistent trend of increasing ejecta mass with increasing impact velocity. When an ejecta blanket is produced though, we can see that higher impact velocities result in higher ejected masses for both quartz sand and JSC-1 targets.

\section{Discussion \\ Observing ejecta}

Due to the hyper- $g$ levels experienced after the low-gravity phase, the state of the target and projectile after each impact is destroyed and the PRIME and COLLIDE data collection relies solely on video recordings. In particular, it is not possible to measure the ejected mass, nor the trajectories of all ejected particles in the case of an ejecta blanket forming. As described in "Ejecta production" section, ejected particles could only be tracked in thin parts of the ejecta blanket or in the case of single particles being lifted rather than a blanket. Figure 11a shows an example of particle tracks superposed with the ejecta blanket: even though we can get particle speeds from these partial tracks, there is a high uncertainty in placing the origin of each track with respect to the surface of the target material and embedded projectile, which location can only be estimated as it is obscured by the ejecta blanket. For this reason, it is not possible to generate mass vs. speed or speed vs. launch position diagrams for ejecta, in order to compare them to 1- $g$ data and the scaling laws derived in Housen and Holsapple (2011).

\section{Observing projectile rebound}

As described in the previous section, a rebound of the projectile from the target was never observed in reducedgravity conditions, compared to microgravity. This could 


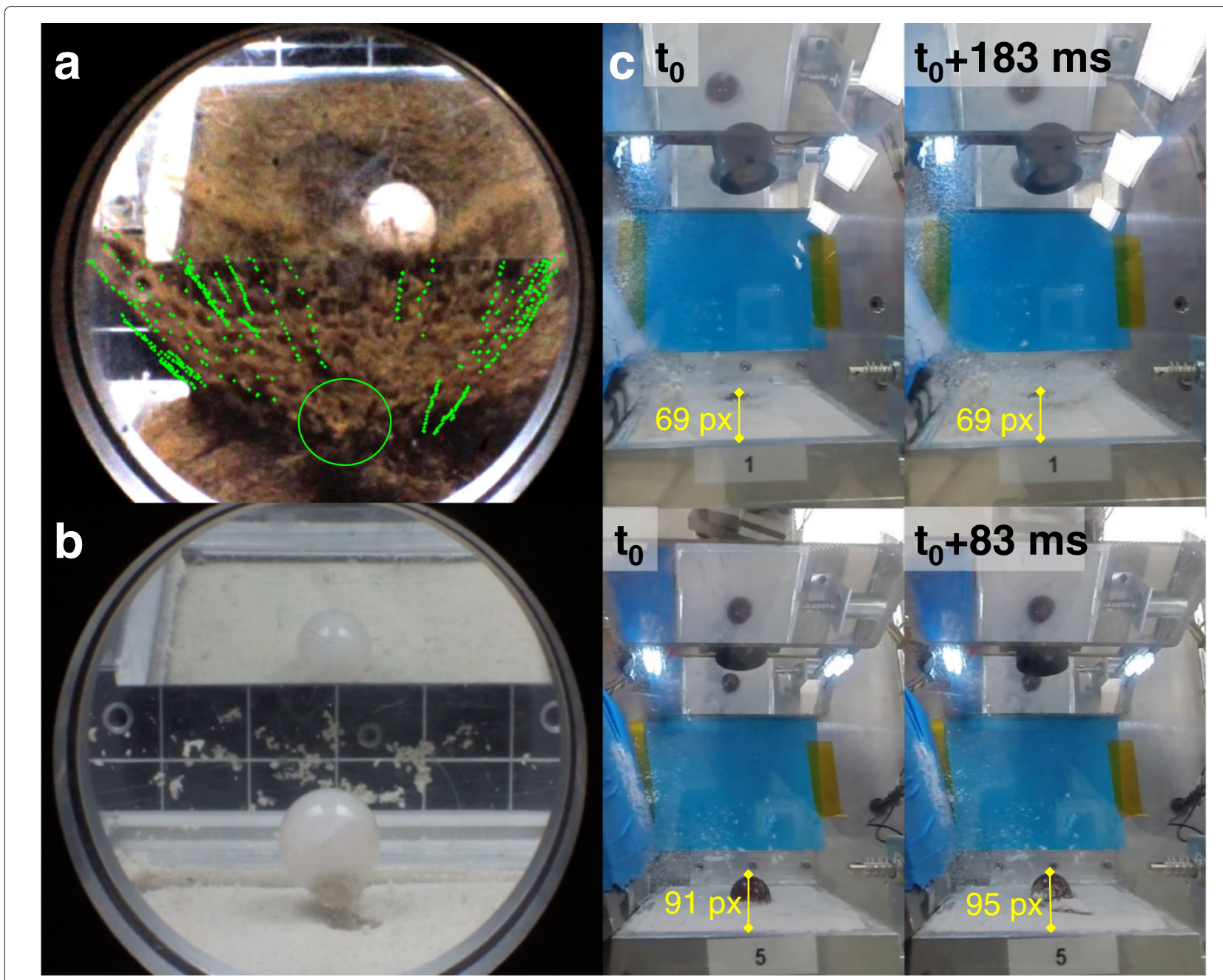

Fig. 11 Data analysis examples. a COLLIDE-3 IBS 3 impact into JSC Mars-1 at $29.3 \mathrm{~cm} / \mathrm{s}$. Particle tracks are superposed in green, and the approximate projectile position is shown with a green circle, as the ejecta curtain hides it entirely. The mirror above the target tray shows a top view of the impact, in which the projectile is visible. b COLLIDE-3 IBS 2 impact into quartz sand at $26.3 \mathrm{~cm} / \mathrm{s}$. Note the mass transfer onto the projectile after rebound. c PRIME-3 rebound observation: $t_{0}$ indicates the moment of deepest penetration of the projectile into the target. (Top) Case of ejecta production without projectile rebound (flight 1, box 1): no marble motion can be detected for $183 \mathrm{~ms}$. In the following frames, the ejecta curtain hides the projectile, which cannot be tracked anymore. (Bottom) Case of ejecta production with projectile rebound (flight 2, box 5): we can track the motion of the projectile over 10 frames and 4 pixels

be due to two factors: either no rebound took place or the rebound was too small to be observed at the space and time resolution of the video recordings of the impacts. In the case that the absence of observed rebound in reduced gravity is only due to resolution limitations of the experiment cameras, we can assume that the rebound takes place with the same coefficient of restitution as in microgravity and calculate the distance and time travelled by the projectile after the impact before it gets stopped by gravity. As the only force on the projectile after it leaves the target again is gravity, simple Newtonian mechanics give us the time $t_{0}$ at the apogee of its trajectory and the height $h$ above the target it reaches at $t_{0}$ :

$$
t_{0}=\frac{v_{r}}{g_{l}} \quad ; \quad h=\frac{1}{2} \frac{v_{r}^{2}}{g_{l}}
$$

where $v_{r}$ is the rebound speed and $g_{l}$ the value of the local gravity acceleration.

For impact speeds around $40 \mathrm{~cm} / \mathrm{s}$, we measure coefficients of restitution around 0.02 (see Fig. 8). This would set $v_{r}$ to $8 \mathrm{~mm} / \mathrm{s}$. Reduced-gravity environments had gravity levels around $10^{-2} g$; thus, $g_{l} \sim 0.1 \mathrm{~m} / \mathrm{s}^{2}$. This leads to a travel time of $t_{0}=80 \mathrm{~ms}$ and a rebound travel height 
of $h=320 \mu \mathrm{m}$. During the PRIME-3 campaign, the camera resolution was around $80 \mu \mathrm{m} /$ pixel and the recording was performed at $120 \mathrm{fps}$ (about $8 \mathrm{~ms}$ between frames). In addition, reduced-gravity environments during this campaign lead to short parabolic trajectories of the ejected particles consistent with a $\sim 0.05 g$ acceleration environment, leaving a clear view of the projectile during the entire recording. This means that a rebound would have been observed and a rebound motion could have been measured for 10 frames and over 4 pixels. As no rebound was observed, it appears that they were due to a different behavior of the target and no rebound actually took place in reduced gravity. To illustrate this, we show two PRIME-3 impacts with different outcomes in Fig. 11c and their measured projectile positions, at deepest penetration $\left(t_{0}\right)$ and after 22 (no rebound) and 4 (rebound) frames. Some impacts generating few ejecta allow for a long-term tracking of the projectile after the impact, allowing for the detection of rebound speeds as low as a few millimeters per second. However, large ejecta blankets obstruct the view of the projectile (see Fig. 11a), thus limiting the observation of very low rebound speeds in these cases.

\section{Scaling the penetration depth}

As shown in Fig. 9, scaling the maximum penetration depth of the projectile into the target $\left(z_{\max }\right)$ with either the impact energy or momentum was not possible. Here, the difference in gravity levels (reduced or microgravity) did not appear in the distribution of the data points. Following Katsuragi and Durian (2013), Fig. 12a shows $z_{\max }$ as a function of the quantity $\mu^{-1}\left(\rho_{p} / \rho_{g}\right)^{1 / 2} D_{p}^{2 / 3} H^{1 / 3}$. In this quantity, $\mu$ is the coefficient of friction of the target material. From angle of repose measurements, we can estimate that $\mu=0.84$ for JSC-1 and 0.67 for quartz sand (Brisset et al. 2016). $\rho_{p}$ and $\rho_{g}$ are the densities of the projectile and target material, respectively; $D_{p}$ is the projectile diameter; and $H$ is the equivalent total drop distance as defined in the paragraph on penetration depth results. Figure 12a allowed us to recognize two groups of data points, which we distinguished by color: impacts with projectiles of masses around $10 \mathrm{~g}$ (see the PRIME-3 data in Table 1: 9.82 to $11.76 \mathrm{~g}$ ) in black and impacts with $\sim 30$ g projectiles (28.2 to $30.75 \mathrm{~g}$ ). Power law fits to each population yielded similar indexes of about 2.5 and a factor of about 3 between both. We concluded that our data set scales with $\mu^{-1}\left(\rho_{p} / \rho_{g}\right)^{0.1} D_{p}^{2 / 3} H^{1 / 3}$, the exponent for $\rho_{p} / \rho_{g}$ being 0.1 rather than 0.5 (all PRIME-3 projectiles have the same diameter of $2 \mathrm{~cm}$ ). The result of this scaling is shown in Fig. 12b.

This scaling and the associated power law index are in contrast with the results by Katsuragi and Durian (2013). In particular, the contribution of the projectile to target densities ratio is reduced from an index 0.5 to 0.25 , while the projectile diameter and impact energy have increased contributions from indexes of 0.67 to 1.67 and 0.33 to 0.83 , respectively. The coefficient of friction has an increased contribution from an index -1 to $-2.5(\mu<1)$. This means that in low gravity (no distinction between reduced gravity and microgravity), the diameter and kinetic energy of the projectiles play a more significant role in the penetration depth than the density ratio with the target material than in $1 g$. This reflects the fact that the gravity pull
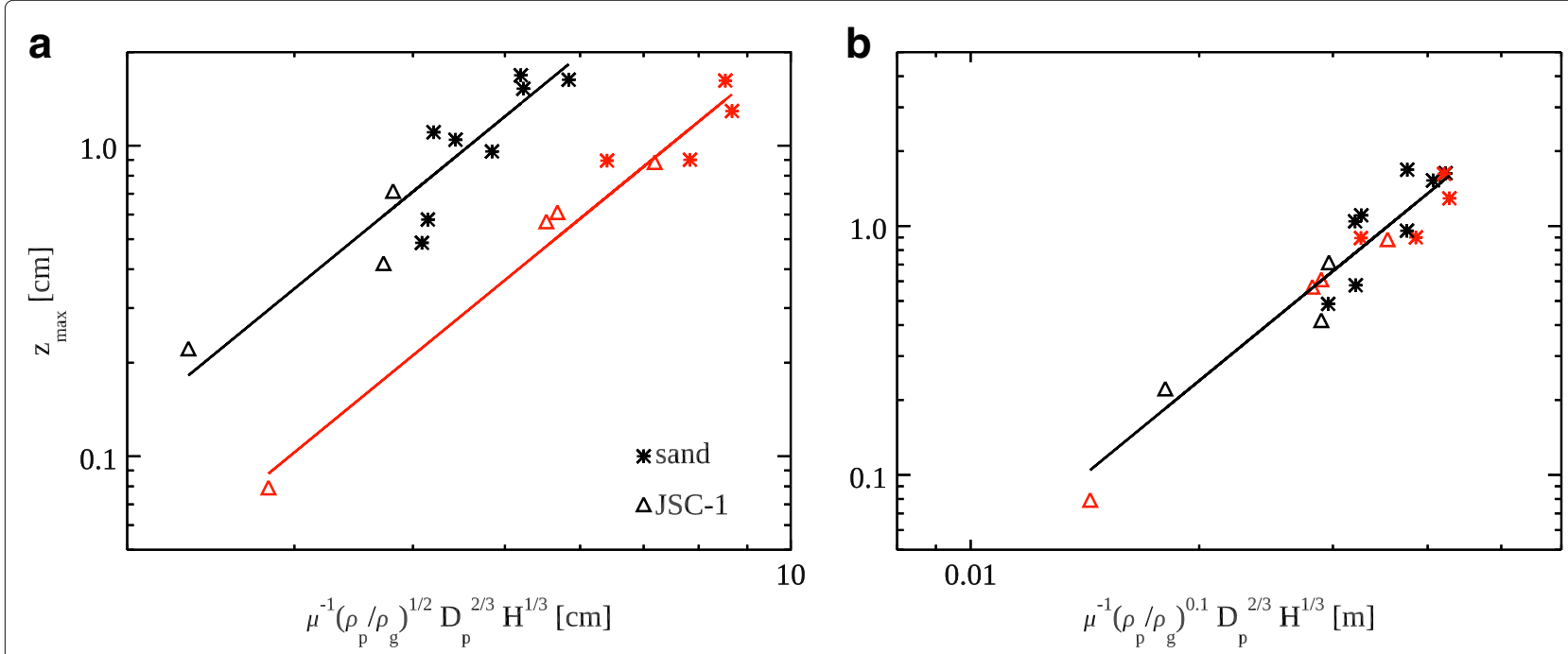

Fig. 12 Scaling of the maximum penetration depth during PRIME-3 impacts. a With the quantity $\mu^{-1}\left(\rho_{p} / \rho_{g}\right)^{1 / 2} D_{p}^{2 / 3} H^{1 / 3}$ (see text for details) and b with $\mu^{-1}\left(\rho_{p} / \rho_{g}\right)^{0.1} D_{p}^{2 / 3} H^{1 / 3}$. Asterisks and triangles represent quartz sand and JSC-1 targets, respectively. Impacts performed with projectiles of masses $\sim 10 \mathrm{~g}$ are marked by black symbols, and $\sim 30 \mathrm{~g}$ by red symbols. The black and red lines in $\mathbf{a}$ are power law fits to these two types of impacts (10 g and $30 \mathrm{~g}$ projectiles), both with indexes $\sim 2.5$. The line in $\mathbf{b}$ shows the power law fit to the entire data set, with an index of 2.5 
on the projectile during penetration is much reduced, and the density ratio between projectile and target material is a less significant factor during penetration. Large and high-velocity projectiles will penetrate the target easier in reduced gravity. The role of the cohesion between the target grains (which is implicit in the coefficient for friction $\mu$ ) is increased by the reduced-gravity environment: cohesive targets are more efficiently stopping a projectile than in $1 g$. This supports the notion that cohesive forces can become significantly more important in low gravity compared to the weight of the target particles (see following paragraph).

\section{Gravity-cohesion competition}

In Fig. 13, we compare the ambient gravity forces to interparticle cohesion forces (van der Waals), following the example of Scheeres et al. (2010), using measured interparticle force values rather than model estimations. The straight black lines in this figure represent the cohesion force between two grains, which is linearly proportional to the particle radius, as measured for two different types of particles. We are using the Johnson-Kendall-Roberts theory (Johnson et al. 1971), estimating the force between two grains to be the pull-off force, $F_{p o}=3 \pi \gamma r, \gamma$ being the surface energy of a grain and $r$ its radius. The surface energies for quartz sand was measured in Kendall et al. (1987) and in Brisset et al. (2016) for polydisperse $\mathrm{SiO}_{2}$ particles in similar experiment conditions (vacuum levels of around 10 mTorr at room temperature). These polydisperse $\mathrm{SiO}_{2}$ grains were similar to JSC-1 lunar simulant in shape, while quartz sand has more rounded, less cohesive grains. The red dashed lines mark the force of gravity on individual grains for three $g$-levels, and the green vertical line marks the largest particles present in the target materials used in the present work.

In this figure, we can see that, for quartz sand, the forces induced on the target grains due to gravity are several orders of magnitude larger than inter-particle adhesive

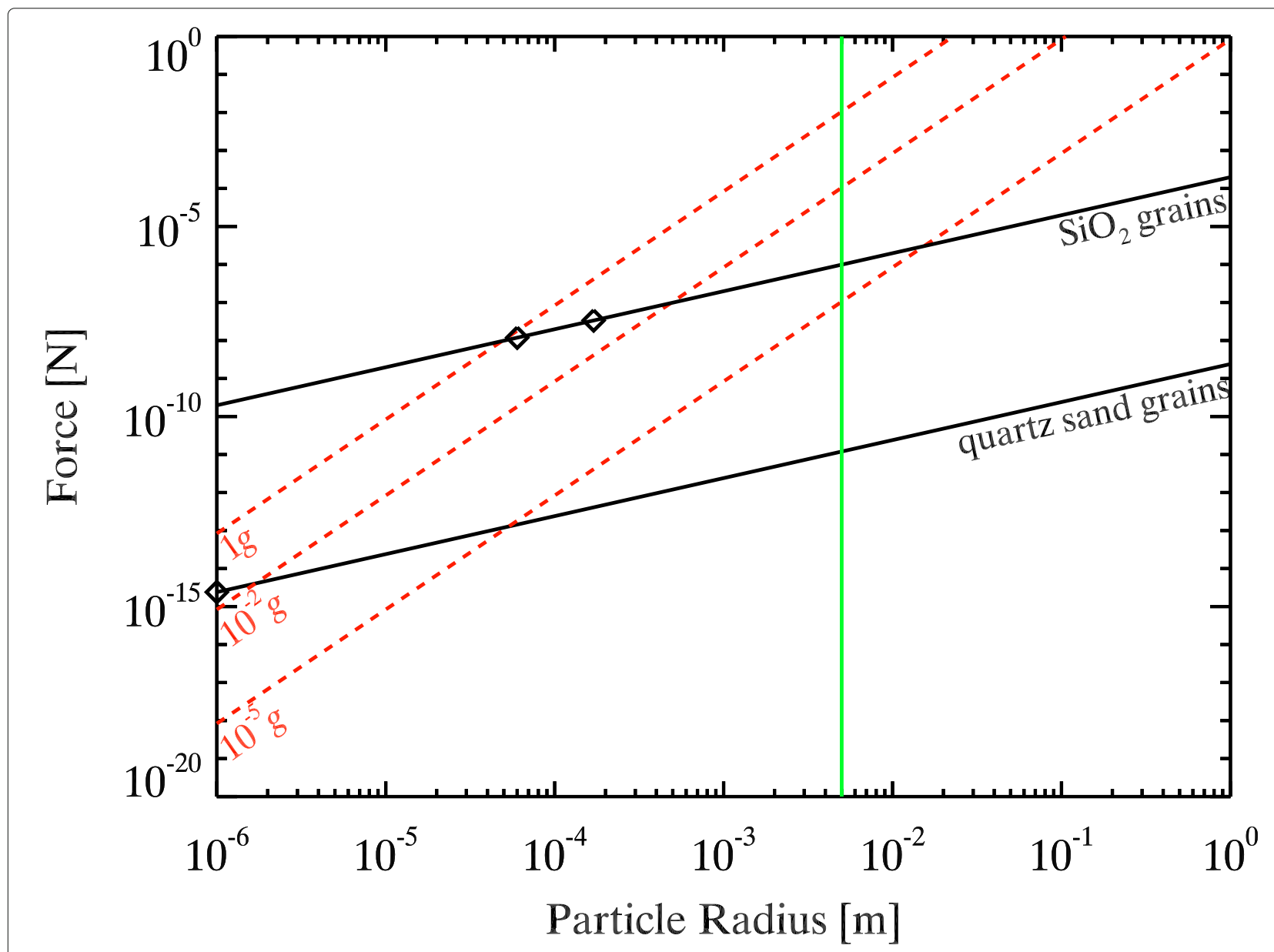

Fig. 13 Comparison between gravitational and cohesive forces depending on the grain radius. The black lines correspond to the measured pull-off force between $\mathrm{SiO}_{2}$ polydisperse (Brisset et al. 2016) and quartz sand grains (Kendall et al. 1987). The grain sizes at which the measurements were performed are marked as diamond symbols. The dashed red lines show the gravitational forces on the regolith grains for different $g$-level environments. The green line marks the largest particles of our target size distributions 
forces at $1 g$ and $10^{-2} g$. Only at $10^{-5} g$, these two forces are of the same order for the smaller target particles. This can explain the reason for lower ejected masses and larger average ejecta speeds (at the same impact speed) observed in reduced gravity compared to microgravity: in reduced gravity, only the fastest, lighter grains can overcome the gravity field and be ejected from the target. In microgravity, the gravity force is low enough to allow for most grains to get lifted, even the slower ones, and the average ejecta speed is accordingly lower. In addition, as the gravity and cohesion forces are of the same order in microgravity, the target material displays a more elastic behavior: cohesion forces between the particles seem to allow for a partially elastic response of the surface to the impact, thus leading to a rebound of the projectile (see Fig. 5, open triangles and diamonds), while in reduced-gravity environments, the presence of a constant downward force locks the particles in place (e.g., jamming; (Cates et al. 1998)) and increases the inter-particle forces due to friction inside the material (Murdoch et al. 2013), thus preventing projectile rebound.

In Fig. 13, we can also see that JSC-1 targets (which approximately behave like $\mathrm{SiO}_{2}$ grains) have stronger cohesion forces between grains than quartz sand: the cohesion and gravity forces are of the same order already in reduced gravity, while cohesion dominates by several orders of magnitude in microgravity. The strong dominance of the cohesive forces can explain why none of the impacts in JSC-1 in microgravity created an ejecta curtain. While most impacts in JSC-1 were performed at speeds below $40 \mathrm{~cm} / \mathrm{s}$ and higher impact speeds may have possibly triggered larger ejecta masses, impacts at similar speeds and in the same microgravity environment generated an ejecta curtain with sand target (Fig. 10). The predominance of cohesive forces between JSC-1 grains kept the target material from escaping.

We can also see a difference in behavior of the JSC-1 targets in reduced-and microgravity environments: for the same impact velocities, rebound is observed in microgravity, while the projectile embeds into the target in reduced gravity (filled black diamonds and red circles in Fig. 5). Even though JSC-1 displays a more cohesive behavior than sand in reduced gravity, the data indicates that these cohesion forces do not dominate gravity enough at $10^{-2} g$ to lead to the rebound of the projectile.

\section{Interacting with small body surfaces}

The experiments presented here are limited by the size of the target tray and projectile. However, it is possible to learn from their results about possible outcomes of surface interactions by spacecraft landers or sampling mechanisms. In particular, impact speeds under $1 \mathrm{~m} / \mathrm{s}$ are typical for past and planned missions interacting with the surface of a small body; Philae touched down on the surface of 67P/Churyumov-Gerasimenko for the first time at $\sim 1 \mathrm{~m} / \mathrm{s}$, OSIRIS-REx's Touch-And-Go (TAG) mechanism is planned to impact the surface of Bennu at around $20 \mathrm{~cm} / \mathrm{s}$, and Hayabusa-2's MASCOT will land on Ryugu also at several $10 \mathrm{~cm} / \mathrm{s}$. The present experiments are therefore in a relevant impact speed regime. While the regolith size distribution on asteroid surfaces seems to heterogeneously include large and fine particles, images of the surfaces of asteroids visited by spacecraft show the presence of regions of very smooth terrain covered in grains of sizes below a millimeter (e.g., the smooth ponds on Eros; Cheng et al. (2002)). These smooth terrains also represent ideal surface interaction sites as they reduce the risks related to landing on uneven surfaces. Therefore, the target size distributions of our experiments are relevant to understanding spacecraft interactions with asteroid surfaces.

At these relevant impact speeds and grain sizes, we observed the new phenomenon of projectile bouncing off of granular material surfaces in microgravity. While numerical simulations are currently the only way of studying low-velocity impacts of spacecraft landers at the correct size scales, those simulations are mostly using hyper-velocity or $1 g$ experiment impact data for calibration. The only simulations predicting and reproducing projectile bouncing from the surface (Maurel et al. 2017), for example, are using Earth gravity impact experiments at $>10 \mathrm{~m} / \mathrm{s}$ into granular material (Yamamoto et al. 2006) or impacts on hard surfaces as input parameters (Biele et al. 2017). Maurel et al. (2017) specifically indicate the need for parameter refinement based on microgravity data from experiments.

It can be noted that the experimental conditions, in particular the level of residual accelerations, have a significant impact on the target behavior. Even for irregular grains that behave like cohesive powders, the granular material displayed a different behavior in reduced gravity and microgravity. This means that the behavior of granular material at the $g$-levels present on the surface of small asteroids $\left(10^{-4} g\right.$ and below) cannot be extrapolated from its behavior at lunar $g$-level $\left(\sim 10^{-2} g\right)$. Therefore, experiments relevant to the interaction with surfaces of bodies like Ryugu and Bennu will require the high-quality microgravity environment of free-fall drop towers or free-floating experiment hardware.

\section{Conclusions}

We have combined and analyzed low-velocity impacts into regolith from five experimental campaigns. The PRIME and COLLIDE experiment setups allowed for recording impacts of centimeter-sized spherical projectiles into a bed of regolith simulant (quartz sand and JSC-1 lunar and JSC Mars-1 simulants), sieved to sizes under $250 \mu \mathrm{m}$. Due to their flight platform, these 
experiments were run either at reduced-gravity levels of $\sim 10^{-2} \mathrm{~g}$ when fixed to the parabolic aircraft or during $0.05 \mathrm{~g}$ parabolas, or at microgravity levels of $<10^{-4} \mathrm{~g}$, when free-floating in the airplane or during Shuttle and suborbital rocket campaigns. The impacts into regolith simulant resulted into four types of outcomes, some producing ejecta and some displaying projectile rebound. These results revealed major differences in the target behavior depending on if the experiment was run in reduced- or microgravity conditions. The main results of the data analysis can be summarized as follows:

- We observed projectile rebound off the target in microgravity. None of the impacts in reduced gravity displayed a similar behavior. The coefficient of restitution of the impacts varied with the impact energy following a power law of index $-1 / 4$ (Fig. 8);

- The maximum penetration depth was observed to depend more on the projectile size and energy and less on its density difference with the target than in $1 g$;

- For the same impact speeds, more mass was ejected in microgravity compared to reduced gravity, but the average ejecta speed was lower (Figs. 6 and 10);

- The difference in cohesive forces in quartz sand and JSC-1 could be observed from the experiment results, with quartz sand behaving like a cohesionless material and JSC-1 displaying cohesive behavior. None of the impacts into JSC-1 generated an ejecta curtain in microgravity, and most of them displayed projectile rebound.

The results of these flight campaigns show that the acceleration environment at which experiments are performed has a significant influence on their outcome. The study of regolith behavior at the surface of small bodies ( $<1 \mathrm{~km}$-sized asteroids) will require acceleration environments below $10^{-4} \mathrm{~g}$, as data cannot be extrapolated from behavior in reduced-gravity environments $\left(>10^{-3} g\right)$.

From our current data set, it clearly appears that more low-velocity impact data is required in microgravity conditions. This is due to the fact that clean microgravity environments are difficult to obtain, with most platforms retaining residual acceleration levels around $10^{-2} \mathrm{~g}$. In addition, more data is currently available for sand targets than for JSC-1 lunar simulant. As JSC-1 is more representative of the granular material expected on asteroids (in particular due to the irregular particle shape), data on JSC-1 targets is more relevant for application to small body surfaces. We can also note that all experiments presented here were run using similar target size distribution: particles sieved to sizes $<250 \mu \mathrm{m}$. From images returned of asteroid Itokawa (Fujiwara et al. 2006) and spectral analysis of Ryugu, we can infer that some asteroid surface regoliths have larger average grain sizes. For a future contribution of our experimental results into small body surface exploration missions, the investigation of grain behavior at sizes between $1 \mathrm{~mm}$ and $1 \mathrm{~cm}$ will be necessary. Such future experiments will be even more sensitive to the quality of the microgravity environment they will be run in due to the larger grain sizes (see Fig. 13).

\section{Abbreviations \\ CMR: Center for Microgravity Research; COLLIDE: COLLisions Into Dust Experiment; IBS: Impactor Box System; ISRU: In situ resource utilization; ISC: Johnson Space Center; MASCOT: Mobile Asteroid Surface Scout; NASA: National Aeronautics and Space Administration; OSIRIS-REx: Origins, Spectral Interpretation, Resource Identification, Security, Regolith Explorer; PRIME: Physics of Regolith Impacts in Microgravity Experiment; TAG: Touch-And-Go}

\section{Acknowledgements}

We would like to thank our two anonymous reviewers for their valuable input and comments.

\section{Funding}

This research is based in part upon work supported by the National Aeronautics and Space Administration under Grant No. NNX11AQ87G issued by the Planetary Geology and Geophysics Program and under grant NNX12AK43G issued by the Outer Planets Research Program, by the NASA Flight Opportunities Program, and by funding from Space Florida for the Center for Microgravity Research.

\section{Availability of data and materials}

Please contact author for data requests.

\section{Authors' contributions}

$J C$ secured funding for all performed experiments and provided the COLLIDE- 1 and COLLIDE-2, and PRIME-1 data. JC, AD, and JB performed the experimental data collection during the PRIME-3 and COLLIDE-3 campaigns. JB, SA, CC, and NM worked on the data analysis. All authors read and approved the final manuscript.

\section{Competing interests}

The authors declare that they have no competing interests.

\section{Publisher's Note}

Springer Nature remains neutral with regard to jurisdictional claims in published maps and institutional affiliations.

Received: 31 October 2017 Accepted: 24 September 2018

Published online: 13 November 2018

\section{References}

Biele J, Ulamec S, Maibaum M, Roll R, Witte L, Jurado E, Muñoz P, Arnold W, Auster H-U, Casas C, Faber C, Fantinati C, Finke F, Fischer H-H, Geurts K, Güttler C, Heinisch P, Herique A, Hviid S, Kargl G, Knapmeyer M, Knollenberg J, Kofman W, Kömle N, Kührt E, Lommatsch V, Mottola S, Pardo de Santayana R, Remetean E, Scholten F, Seidensticker KJ, Sierks H Spohn T (2015) The landing(s) of Philae and inferences about comet surface mechanical properties. Science 349(1). https://doi.org/10.1126/ science.aaa9816

Biele J, Kesseler L, Grimm CD, Schröder S, Mierheim O, Lange M, Ho T-M (2017) Experimental determination of the structural coefficient of restitution of a bouncing asteroid lander. ArXiv preprint arXiv:1705.00701

Blum J, Wurm G (2008) The growth mechanisms of macroscopic bodies in protoplanetary disks. Annu Rev Astron Astrophys 46:21-56. https://doi org/10.1146/annurev.astro.46.060407.145152

Brisset J, Colwell JE, Dove A, Rascon A, Mohammed N, Cox C (2016) Low-velocity impacts into cryogenic icy regolith. In: AAS/Division for 
Planetary Sciences Meeting Abstracts, vol. 48. American Astronomical Society. http://adsabs.harvard.edu/abs/2016DPS....4851805B

Brisset J, Heißelmann D, Kothe S, Weidling R, Blum J (2016) Submillimetre-sized dust aggregate collision and growth properties. Experimental study of a multi-particle system on a suborbital rocket. Astron Astrophys 593:A3. https://doi.org/10.1051/0004-6361/201527288

Britt DT, Yeomans D, Housen K, Consolmagno G (2002) Asteroid density, porosity, and structure p. 485-500

Cates ME, Wittmer JP, Bouchaud J-P, Claudin PH (1998) Jamming, force chains, and fragile matter. Phys Rev Lett 81(9):1841

Cheng AF, Izenberg N, Chapman CR, Zuber MT (2002) Ponded deposits on asteroid 433 Eros. Meteorit Planet Sci 37:1095-1105. https://doi.org/10. 1111/j.1945-5100.2002.tb00880.x

Clark AH, Kondic L, Behringer RP (2012) Particle scale dynamics in granular impact. Phys Rev Lett 109(23):238302. https://doi.org/10.1103/ PhysRevLett.109.238302

Clark AHM, Behringer RP (2013) Granular impact model as an energy-depth relation. EPL (Europhys Lett) 101:64001. https://doi.org/10.1209/0295$5075 / 101 / 64001$

Colwell J, Brisset J, Dove A, Whizin A, Nagler H, Brown N, Rascon A, Brightwell K, Seward L (2016) Low-velocity impacts into regolith under microgravity conditions. In: 15th Biennial ASCE Conference on Engineering, Science, Construction, and Operations in Challenging Environments, volume 82 of Earth and Space Conference. https://ascelibrary.org/doi/10.1061/ 9780784479971.010

Colwell JE (2003) Low velocity impacts into dust: results from the COLLIDE-2 microgravity experiment. Icarus 164:188-196. https://doi.org/10.1016/ S0019-1035(03)00083-6

Colwell JE, Sture S, Cintala M, Durda D, Hendrix A, Goudie T, Curtis D, Ashcom DJ, Kanter M, Keohane T, Lemos A, Lupton M, Route M (2008) Ejecta from impacts at $0.22 .3 \mathrm{~m} / \mathrm{s}$ in low gravity. Icarus 195:908-917. https://doi.org/10.1016/j.icarus.2007.12.019

Colwell JE, Taylor M (1999) Low-velocity microgravity impact experiments into simulated regolith. Icarus 138:241-248. https://doi.org/10.1006/icar.1998. 6073

Deboeuf S, Gondret P, Rabaud M (2009) Dynamics of grain ejection by sphere impact on a granular bed. Phys Rev E 79(4):041306. https://doi.org/10. 1103/PhysRevE.79.041306

Dombard AJ, Barnouin OS, Prockter LM, Thomas PC (2010) Boulders and ponds on the Asteroid 433 Eros. Icarus 210:713-721. https://doi.org/10.1016/j. icarus.2010.07.006

Fujiwara A, Kawaguchi J, Yeomans DK, Abe M, Mukai T, Okada T, Saito J, Yano H, Yoshikawa M, Scheeres DJ, Barnouin-Jha O, Cheng AF, Demura H, Gaskell RW, Hirata N, Ikeda H, Kominato T, Miyamoto H, Nakamura AM, Nakamura R, Sasaki S, Uesugi K (2006) The rubble-pile asteroid Itokawa as observed by Hayabusa. Science 312:1330-1334. https://doi.org/10.1126/ science. 1125841

Gundlach B, Blum J (2013) A new method to determine the grain size of planetary regolith. Icarus 223:479-492. https://doi.org/10.1016/j.icarus. 2012.11.039

Güttler C, Krause M, Geretshauser RJ, Speith R, Blum J (2009) The physics of protoplanetesimal dust agglomerates. IV. Toward a dynamical collision model. Astrophys J 701:130-141. https://doi.org/10.1088/0004-637X/701/ $1 / 130$

Hirabayashi M, Sánchez DP, Scheeres DJ (2015) Internal structure of asteroids having surface shedding due to rotational instability. Astrophys J 808:63. https://doi.org/10.1088/0004-637X/808/1/63

Hiroi T, Pieters CM, Takeda H (1994) Grain size of the surface regolith of asteroid 4 Vesta estimated from its reflectance spectrum in comparison with HED meteorites. Meteoritics 29:394-396

Holsapple K, Giblin I, Housen K, Nakamura A, Ryan E (2002) Asteroid impacts: laboratory experiments and scaling laws

Housen KR, Holsapple KA (2003) Impact cratering on porous asteroids. Icarus 163:102-119. https://doi.org/10.1016/S0019-1035(03)00024-1

Housen, KR, Holsapple KA (2011) Ejecta from impact craters. Icarus 211:856-875. https://doi.org/10.1016/j.icarus.2010.09.017

Housen, KR, Wilkening LL (1982) Regoliths on small bodies in the solar system. Ann Rev Earth Planet Sci 10:355-376. https://doi.org/10.1146/annurev.ea. 10.050182.002035

Housen KR, Wilkening LL, Chapman CR, Greenberg R (1979) Asteroidal regoliths. Icarus 39:317-351. https://doi.org/10.1016/0019-1035(79)90145-3
Housen KR, Holsapple KA, Voss ME (1999) Compaction as the origin of the unusual craters on the asteroid Mathilde. Nature 402:155-157. https://doi. org/10.1038/45985

Jaumann R, Bibring JP, Glassmeier KH, Grott M, Ho TM, Ulamec S, Schmitz N, Auster HU, Biele J, Kuninaka H, Okada T (2016) A Mobile Asteroid Surface Scout (MASCOT) on board the Hayabusa 2 Mission to the near Earth asteroid (162173) Ryugu. in AGU Fall Meeting Abstracts

Johnson KL, Kendall K, Roberts AD (1971) Surface energy and the contact of elastic solids. Proc R Soc Lond 324:301-313

Johnson KL (1985) Contact mechanics. Cambridge university press, Cambridge

Katsuragi H, Blum J (2017) The physics of protoplanetesimal dust agglomerates. IX. Mechanical properties of dust aggregates probed by a solid-projectile impact. Astrophys J 851:23. https://doi.org/10.3847/1538-4357/aa970d

Katsuragi H, Durian DJ (2007) Unified force law for granular impact cratering. Nat Phys 3:420-423. https://doi.org/10.1038/nphys583

Katsuragi, H, Durian DJ (2013) Drag force scaling for penetration into granular media. Phys Rev E 87(5):052208. https://doi.org/10.1103/PhysRevE.87. 052208

Kawaguchi J, Fujiwara A, Uesugi T (2008) Hayabusa - its technology and science accomplishment summary and Hayabusa-2. Acta Astronaut 62:639-647. https://doi.org/10.1016/j.actaastro.2008.01.028

Kendall K, McN. Alford N, Birchall JD (1987) A new method for measuring the surface energy of solids. Nature 6107:794-796. https://doi.org/10.1038/ $325794 a 0$

Klimek R, Wright R (2006) Spotlight-8 Image Analysis Software. NASA TM 214084 2005. http://microgravity.grc.nasa.gov/spotlight/

Lauretta DS, Balram-Knutson SS, Beshore E, Boynton WV, Drouet d'Aubigny C, DellaGiustina DN, Enos HL, Golish DR, Hergenrother CW, Howell ES, Bennett CA, Morton ET, Nolan MC, Rizk B, Roper HL, Bartels AE, Bos BJ, Dworkin JP, Highsmith DE, Lorenz DA, Lim LF, Mink R, Moreau MC, Nuth JA, Reuter DC, Simon AA, Bierhaus EB, Bryan BH, Ballouz R, Barnouin OS, Binzel RP, Bottke WF, Hamilton VE, Walsh KJ, Chesley SR, Christensen PR, Clark BE, Connolly HC, Crombie MK, Daly MG, Emery JP, McCoy TJ, McMahon JW, Scheeres DJ, Messenger S, Nakamura-Messenger K, Righter K, Sandford SA (2017) OSIRIS-REx: sample return from Asteroid (101955) Bennu. Space Sci Rev 212(1-2):925-984. https://doi.org/10.1007/ s11214-017-0405-1

Machii N, Nakamura AM, Güttler C, Beger D, Blum J (2013) Collision of a chondrule with matrix: relation between static strength of matrix and impact pressure. Icarus 226:111-118. https://doi.org/10.1016/j.icarus.2013. 05.006

Maurel C, Michel P, Biele J, Ballouz R-L, Thuillet F (2017) Numerical simulations of the contact between the lander mascot and a regolith-covered surface. Adv Space Res 62(8):2099-2124

Murdoch N, Rozitis B, Nordstrom K, Green SF, Michel P, de Lophem T-L, Losert W (2013) Granular convection in microgravity. Phys Rev Lett 110(1):018307. https://doi.org/10.1103/PhysRevLett.110.018307

Sanchez P, Scheeres DJ (2014) The strength of regolith and rubble pile asteroids. Meteorit Planet Sci 49:788-811. https://doi.org/10.1111/maps. 12293

Sánchez P, Scheeres DJ, Bierhaus EB, Clark B (2013) Regolith penetrometry in microgravity. Eur Planet Sci Congr 8:EPSC2013-882

Scheeres DJ, Hartzell CM, Sánchez P, Swift M (2010) Scaling forces to asteroid surfaces: the role of cohesion. Icarus 210:968-984. https://doi.org/10.1016/ j.icarus.2010.07.009

Seguin A, Bertho Y, Gondret P (2008) Influence of confinement on granular penetration by impact. Phys Rev E 78(1):010301. https://doi.org/10.1103/ PhysRevE.78.010301

Thornton C, Ning Z (1998) A theoretical model for the stick/bounce behaviour of adhesive, elastic-plastic spheres. Powder Technol 99(2):154-162

Thuillet F, Maurel C, Michel P, Biele J, Ballouz R-L, Richardson DC (2017) Numerical simulations of surface package landing on a low-gravity granular surface: application to the landing of MASCOT onboard Hayabusa2. In: Lunar and Planetary Science Conference, Vol. 48. 48th Lunar and Planetary Science Conference. https://www.hou.usra.edu/meetings/ Ipsc2017/pdf/1810.pdf

Tsuda Y, Yoshikawa M, Abe M, Minamino H, Nakazawa S (2013) System design of the Hayabusa 2 - asteroid sample return mission to 1999 JU3. Acta Astronaut 91:356-362. https://doi.org/10.1016/j.actaastro.2013.06.028

Veverka J, Farquhar B, Robinson M, Thomas P, Murchie S, Harch A, Antreasian PG, Chesley SR, Miller JK, Owen WM, Williams BG, Yeomans D, 
Dunham D, Heyler G, Holdridge M, Nelson RL, Whittenburg KE, Ray JC, Carcich B, Cheng A, Chapman C, Bell JF, Bell M, Bussey B, Clark B, Domingue D, Gaffey MJ, Hawkins E, Izenberg N, Joseph J, Kirk R, Lucey P, Malin M, McFadden L, Merline WJ, Peterson C, Prockter L, Warren J, Wellnitz D (2001) The landing of the NEAR-Shoemaker spacecraft on asteroid 433 Eros. Nature 413:390-393. https://doi.org/10.1038/35096507

Wagner E, DeForest CE (2016) Opportunities for suborbital space and atmospheric research facilities on Blue Origin's New Shepard crew capsule. AGU Fall Meeting Abstracts P53C-2216. http://adsabs.harvard.edu/abs/ 2016AGUFM.P53C2216W

Yamamoto S, Wada K, Okabe N, Matsui T (2006) Transient crater growth in granular targets: an experimental study of low velocity impacts into glass sphere targets. Icarus 183:215-224. https://doi.org/10.1016/j.icarus.2006. 02.002

\section{Submit your manuscript to a SpringerOpen ${ }^{\circ}$ journal and benefit from:}

- Convenient online submission

- Rigorous peer review

- Open access: articles freely available online

- High visibility within the field

- Retaining the copyright to your article

Submit your next manuscript at $\gg$ springeropen.com 